\title{
OPEN Cobalamin is present in cells of non-tuberculous mycobacteria, but not in Mycobacterium tuberculosis
}

\begin{abstract}
Alina Minias $^{1 \bowtie}$, Filip Gąsior ${ }^{1,2}$, Anna Brzostek ${ }^{1}$, Tomasz Jagielski \& Jarosław Dziadek ${ }^{1 \bowtie}$
Cobalamin (vitamin B12) is a structurally complex molecule that acts as a cofactor for enzymes and regulates gene expression through so-called riboswitches. The existing literature on the vitamin B12 synthesis capacity in Mycobacterium tuberculosis is ambiguous, while in non-tuberculous mycobacteria (NTM) is rather marginal. Here we present the results of our investigation into the occurrence of vitamin B12 in mycobacteria. For detection purposes, immunoassay methods were applied to cell lysates of NTM and $M$. tuberculosis clinical and laboratory strains grown under different conditions. We show that whereas vitamin B12 is present in cells of various NTM species, it cannot be evidenced in strains of differently cultured $M$. tuberculosis, even though the genes responsible for vitamin B12 synthesis are actively expressed based on RNA-Seq data. In summary, we conclude that the production of vitamin B12 does occur in mycobacteria, with the likely exception of $M$. tuberculosis. Our results provide direct evidence of vitamin B12 synthesis in a clinically important group of bacteria.
\end{abstract}

Cobalamin (vitamin B12) is a structurally complex molecule consisting of four linked pyrrole rings and the cobalt ion in the center. There are four chemical forms of cobalamin that differ in the upper ligand: hydroxocobalamin $\left(\mathrm{OHB}_{12}\right)$, methylcobalamin $\left(\mathrm{CH}_{3} \mathrm{~B}_{12}\right)$, deoxyadenosylcobalamin $\left(\mathrm{AdoB}_{12}\right)$, and most chemically stable, cyanocobalamin $\left(\mathrm{CNB}_{12}\right)$.

The chemical synthesis of cobalamin involves approximately 70 reactions. Microbial synthesis, which can be aerobic or anaerobic, involves fewer steps (Fig. 1). De novo synthesis involves about 30 reactions starting from glutamate. The salvage pathway is shorter than de novo synthesis, and it involves 12 genes ${ }^{1,2}$. Pseudomonas denitrificans, Propionibacterium shermanii, Sinorhizobium meliloti, Eschericha coli and Bacillus megaterium are the main producers of $\mathrm{CNB}_{12}$ at the industrial scale ${ }^{1}$. Organisms that use vitamin B12 in their metabolism, and at the same time do not have the gene repertoire enabling its biosynthesis, use exogenous cobalamin actively transported through dedicated ABC transporters ${ }^{3,4}$.

Cobalamin influences cell metabolism via two mechanisms. It acts as a cofactor for enzymes, and regulates gene expression through so-called riboswitches. There are three major subfamilies of vitamin B12-dependent enzymes: $\mathrm{AdoB}_{12}$-dependent isomerases, $\mathrm{CH}_{3} \mathrm{~B}_{12}$-dependent methyltransferases, and dehalogenases. The isomerases are the largest subfamily of B12-dependent enzymes. They play important roles in fermentation pathways. An example of B12-dependent isomerases is methylmalonyl-CoA mutase (MCM), found in bacteria and humans. Together with methylmalonyl-CoA epimerase, the enzyme is involved in converting propionate to succinate through the methylmalonyl-CoA pathway. Here, the enzyme catalyzes the reversible isomerization of l-methylmalonyl-CoA to succinyl-CoA using AdoCbl as a cofactor. Another common B12-dependent isomerase is ribonucleotide reductase (NrdZ). The enzyme catalyzes the conversion of ribonucleotides to deoxyribonucleotides for DNA replication and repair. AdoCbl adenosyl ribose is required to allow hydrogen transfer to the catalytic thiol group ${ }^{5}$. The B12-dependent methyltransferases play an important role in amino acid metabolism and $\mathrm{CO}_{2}$ fixation in anaerobic microorganisms. The most extensively studied B12-dependent methyltransferase is methionine synthase $(\mathrm{MetH})$. This enzyme is responsible for the regeneration of methionine from homocysteine via the vitamin B12-dependent pathway and is involved in the folate pathway (Fig. 2). The methyl group of methylcobalamin is transferred to homocysteine forming methionine ${ }^{6}$. Vitamin B12-dependent dehalogenases

${ }^{1}$ Laboratory of Genetics and Physiology of Mycobacterium, Institute of Medical Biology, Polish Academy of Sciences, Lodz, Poland. 'BioMedChem Doctoral School of the University of Lodz and the Institutes of the Polish Academy of Sciences in Lodz, Lodz, Poland. ${ }^{3}$ Department of Medical Microbiology, Institute of Microbiology, Faculty of Biology, University of Warsaw, Warsaw, Poland. ${ }^{\varpi}$ email: alinagorna@gmail.com; jdziadek@cbm.pan.pl 




Figure 1. Synthesis of vitamin B12 in bacteria (figure adapted with permission from https://www.ncbi.nlm. nih.gov/pmc/articles/PMC5282855/ under the terms of the Creative Commons Attribution 4.0 International License).

are present in anaerobic bacteria. Reductive dehalogenases have a vital role in detoxifying aromatic and aliphatic chlorinated organic compounds ${ }^{7}$.

Riboswitches were first detected in E. coli (Nou and Kadner, 1998). They are metabolite binding domains in specific mRNAs. Although riboswitches were mostly identified in prokaryotes, they are also present in higher organisms. They react to changes in the environment, such as changes in temperature, $\mathrm{pH}$, or cofactor presence. Ligand binding allows for allosteric rearrangement of the mRNA and results in post-transcriptional control of gene expression. Cobalamin riboswitches repress gene expression by binding the ligand and preventing the mRNA's binding to ribosomes ${ }^{8}$.

The genus Mycobacterium accommodates bacterial species that carry genes presumably involved in the synthesis of vitamin B12. Mycobacteria are split into five phylogenetic clades, namely "tuberculosis-simiae", "terrae", "triviale", "fortuitum-vaccae", and "abscessus-chelonae". There are several important pathogens in these groups. The most infamous one is M. tuberculosis, a causative agent of tuberculosis. This is an obligatory intracellular human pathogen with a complex life cycle. As shown previously, nearly all genes required for aerobic cobalamin synthesis are identifiable in $M$. tuberculosis, except for the cobF coding for precorin-6a synthase 9 .

Further research on $M$. tuberculosis confirmed the presence of two vitamin B12-dependent riboswitches in its genome, encoded by the Rv1133c and Rv0256c genes ${ }^{10}$. Rv1133c encodes a riboswitch regulating the metE gene expression of the cobalamin-independent methionine synthase (Fig. 2$)^{11}$. The second cobalamin sensitive 


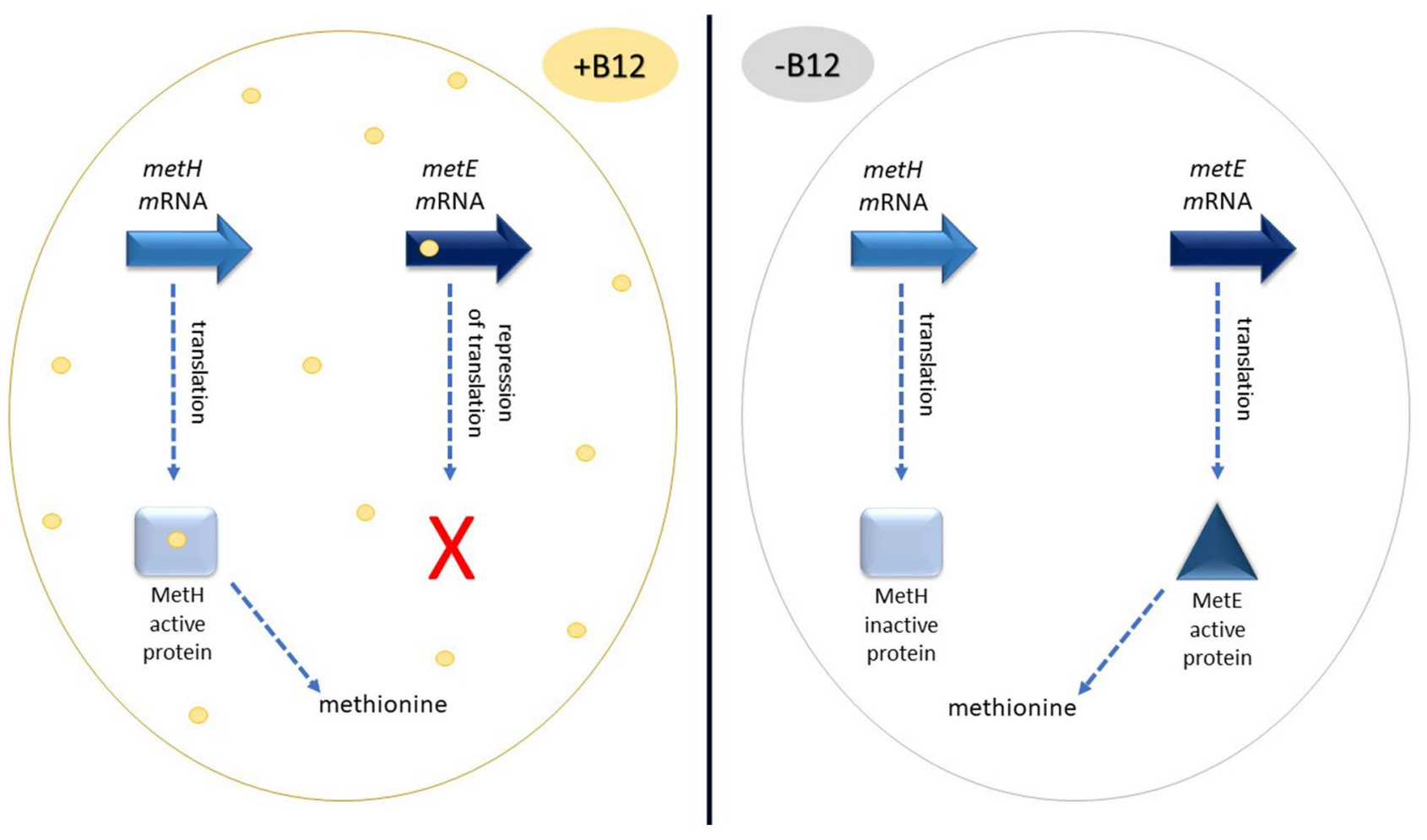

Figure 2. Synthesis of methionine in M. tuberculosis. In the presence of vitamin B12 the riboswitch represses the translation of mRNA of metE. Cobalamin binds as a cofactor to MetH protein, and the latter provides methionine necessary for the cell. In turn, in the absence of vitamin B12, MetH is not functional. metE transcripts are efficiently transcribed to MetE protein, which provides methionine.

riboswitch at Rv0256c affects the PPE2-cobQ1-cobU operon. Rv0256c (PPE2) encodes a PPE2 family protein, while CobQ1 and CobU are presumably involved in vitamin B12 synthesis.

The identification of genes involved in the uptake of vitamin B12 from the environment in M. tuberculosis was performed by random mutagenesis ${ }^{12}$. Deletion of the Rv1819c gene, encoding ABC transporter BacA, abolished the ability to transport vitamin B12. Moreover, deletion of the bacA did not affect the infectivity of tubercle bacilli, albeit virulence was reduced during prolonged infection ${ }^{4}$.

MetH, MutB, and NrdZ are the three cobalamin-dependent proteins of M. tuberculosis. Studies involving these proteins cast doubt whether the reference strain of $M$. tuberculosis H37Rv synthesizes cobalamin. Savvi et al. showed that $M$. tuberculosis H37Rv could not use propionate as a carbon source by using the methylmalonyl pathway without enriching the medium in vitamin B12 ${ }^{13}$. Warner et al. showed that B12 supplementation is necessary for the growth of the $\Delta m e t E$ mutant of $M$. tuberculosis $\mathrm{H} 37 \mathrm{Rv}$, which also requires the MetH cobalamindependent enzyme ${ }^{11}$. Both of these reports suggest that $M$. tuberculosis cannot synthesize cobalamin and relies on cobalamin scavenged from the host. In turn, the M. tuberculosis clinical strain CDC1551 was surmised to be able to synthesize cobalamin by demonstrating a truncated variant of MetH. It depends on MetE to synthesize cobalamin. Therefore, it is hyper susceptible to sulfonamides, which block the folate cycle where MetH is involved in the salvage pathway. When M. tuberculosis CDC1551 was carrying MetH of H37Rv in trans, the authors observed that the strain presented standard susceptibility to sulfonamides. They suspected that intracellular levels of cobalamin in M. tuberculosis CDC1551 allow for activation of $\mathrm{MetH}^{14}$. In 2018, we showed that genes presumably involved in vitamin B12 synthesis and metabolism are under purifying selective pressure, suggesting functionality of pathway ${ }^{15}$. Ignatov et al. showed that genes involved in vitamin B12 synthesis upregulate their expression during mycobacterial persistence, reached by growing bacteria in a medium deprived of $\mathrm{K}+{ }^{16}$. In summary, information regarding the possibility of vitamin B12 synthesis in M. tuberculosis is chaotic. As for other mycobacteria, information is scarce. Vitamin B12 synthesis was confirmed in Mycolicibacterium smegmatis ${ }^{17,18}$. One manuscript, published in 1977, currently not available for a full read online, reported the presence of vitamin B12 in the cells of M. smegmatis, Mycolicibacterium fortuitum, Mycobacterium asiaticum, Mycobacterium phlei, and Mycobacterium bovis BCG using Lactobacillus leichmannii ATCC7830 tube method ${ }^{19}$.

Here we present the results of our investigation on the presence of vitamin B12 in mycobacteria. The purpose of this study was to describe vitamin B12 production in phylogenetic order of Mycobacterium. We show that vitamin B12 is present in the cells of various non-tuberculous species. Interestingly, we could not identify vitamin B12 in several strains of M. tuberculosis cultured under different growth conditions, even though the genes responsible for vitamin B12 synthesis are actively expressed. 


\section{Results and discussion}

Gene expression of vitamin B12 synthesis genes. We aimedto identify the genes involved in vitamin B12 synthesis in NTM included in this study (Table 1). We used whole-genome sequencing data and its annotation found in the major bioinformatics databases. The available data provided an incomplete indication of loci involved in the vitamin B12 biosynthesis pathway, as it is for M. tuberculosis. The precision of annotation, covering the entire extent of variability of proteins serving particular functions, is still to be developed.

We used RNA-Seq data available at ENA Database to estimate gene expression through transcripts per million base pair (TPM) values for genes involved in vitamin B12 synthesis in M. tuberculosis, M. abscessus subsp. abscessus, and M. smegmatis (Table 2). TPM values inform about the level of basal transcription of genes, and are not to be confused with relative gene expression in different conditions. The average gene expression for M. abscessus and M. smegmatis was $201.88 \pm 547.4$ TPM and $147.33 \pm 607.04$ TPM, respectively. In comparison, the average expression of genes predicted to be involved in vitamin B12 synthesis was $94.943 \pm 9.483 \mathrm{TPM}$ and $76.669 \pm 29.645$ TPM, respectively. For M. tuberculosis we investigated gene expression level in cells grown in rich broth $^{20}$, in medium supplemented with cholesterol ${ }^{21}$, and in human macrophages ${ }^{22}$. The above conditions' average gene expression was $256.02 \pm 551.112$ TPM, $256.01 \pm 764.53$ TPM, and 256.02 \pm 1039.71 TPM, respectively. Simultaneously, the average expression of genes predicted to be involved in vitamin B12 aerobic synthesis was lower, 114.114 \pm 77.666 TPM, 54.189 \pm 35.772 TPM, $145.871 \pm 159.664$ TPM, respectively. Their overall expression level was comparable to DnaG primase, an essential protein involved in DNA replication (104.986 \pm 2.321 , $91.056 \pm 42.023$, and $118.236 \pm 98.324$, respectively $)^{23}$.

Studies with Propionibacterium sp. showed the crucial role of cobA gene in regulating the level of synthesis of vitamin B12. Vitamin B12 was shown to regulate the cobA operon through a riboswitch in its $5^{\prime}$ untranslated region (5' UTR $)^{24}$. Similarly, M. tuberculosis contains a PPE2-cobQ1-cobU operon, containing vitamin B12 synthesis genes and controlled by a riboswitch. Taken the ubiquity of vitamin B12 riboswitches across Prokarytotes, the mechanisms where the level of vitamin B12 synthesis genes seem to be controlled by the synthesis product might be common ${ }^{25}$. Presented results show that $c o b Q 1$ and $c o b U$ of $M$. tuberculosis are actively expressed in a rich broth and in the presence of cholesterol. Expression of cobQ1 was not observed in macrophages. The level of reading coverage of the mycobacterial genome is relatively low. We assume that the low coverage results from natural technical difficulties of isolating mycobacterial RNA from the Eukaryotic cells that have RNA of their $\mathrm{own}^{26}$. Since reads of $c o b U$ are present, we suspect that the absence of $c o b Q 1$ reads in macrophages is due to too low coverage.

Vitamin B12 concentration in non-tuberculous mycobacteria. We measured the concentration of vitamin B12 per mg of protein in cell lysates obtained from 7H9 medium cultures of various species of NTM spread across the phylogenetic tree (Fig. 3A $)^{27}$. On average, mycobacterial cells contained $33.044 \mathrm{ng}$ of cobalamin per mg of protein. The median level of vitamin B12 across analyzed cells was $29.217 \mathrm{ng}$ per $\mathrm{mg}$ of protein. The lowest concentration of vitamin B12 was detected for M. innocens ( $3.704 \pm 0.643 \mathrm{ng} / \mathrm{mg}$ of protein). The highest concentration of vitamin B12 was detected in M. attenuatum $(90.211 \pm 13,769 \mathrm{ng} / \mathrm{mg}$ of protein). Results regarding relatively high production of vitamin B12 in M. phlei (77.712 $\pm 10.597 \mathrm{ng} / \mathrm{mg}$ of protein), when compared with other species of mycobacteria, are in line with previous findings from $1977^{19}$. When vitamin B12 concentration was normalized to protein content, we detected a higher concentration of vitamin B12 in mycobacteria than it was previously detected in P. aeruginosa. There, analyses by HPLC-MS detected from 0.32 to $3.72 \mathrm{ng}$ of vitamin B12 per mg of protein, depending on culture conditions and $\operatorname{strain}^{28}$.

There are different approaches to the normalization of vitamin B12 concentration in bacteria. To further compare our results with other bacterial species, we also normalized our data regarding vitamin B12 concentration to $\mathrm{ml}$ of culture (Fig. 3B). When calculated in such a way, we obtained from 0.049 to $1.2 \mathrm{ng}$ of vitamin B12 per $\mathrm{ml}$ of culture. In comparison, Pseudomonas freudenreichii produced from 20 to $125 \mathrm{ng}$ of vitamin B12/ ml of culture, depending on culture condition $\mathrm{s}^{29}$. B. megaterium produced from $0.26 \mathrm{ng} / \mathrm{ml}$ of culture to $204 \mathrm{ng} /$ $\mathrm{ml}$ of culture, also depending on the culture conditions. Due to relatively low concentration of vitamin B12, expensive growth media, long culture time, and difficulties to disrupt the cells, we conclude that mycobacteria are not attractive alternative producers of vitamin B12 at the industrial scale.

Importantly, we show that NTM can produce vitamin B12, and synthesis is shared across the phylogenetic tree. The sensitivity of the immunoassay detection was suitable for the detection of vitamin B12 in mycobacterial cells. This observation is an important reference point for results obtained for M. tuberculosis.

The level of vitamin B12 concentration in the NTM cells is variable, and it depends on the cell line. M. abscessus complex is a group of non-tuberculous mycobacteria. It is an emerging human pathogen often associated with the infection of cystic fibrosis patients. It consists of three subspecies M. abscessus subsp. abscessus, M. abscessus subsp. massiliense and M. abscessus subsp. bolletii. We measured the concentration of vitamin B12 per mg of protein in cell lysates obtained from $7 \mathrm{H} 9$ medium cultures of various clinical strains of M. abscessus subsp. abscessus and M. abscessus subsp. bolletii (Fig. 3C). We detected vitamin B12 in cells of all of the analyzed strains. On average, cells contained $19.842 \mathrm{ng}$ of cobalamin per $\mathrm{mg}$ of protein. The median level of vitamin B12 across analyzed cells was $18.121 \mathrm{ng}$ per $\mathrm{mg}$ of protein. The lowest concentration of vitamin B12 was detected for M. abscessus subsp. abscessus strain A5 (14.861 \pm 1.848$)$. The highest concentration of vitamin B12 was detected in $M$. abscessus subsp. abscessus strain A7 (31.582 \pm 1.071$)$. The difference in concentration between the highest and the lowest producing strain was statistically significant $(p<0.01, \mathrm{t}=11.07, \mathrm{df}=3)$.

We observed up to a twofold difference in the level of vitamin B12 synthesis across distinct strains of the same species. Strain variability in cobalamin concentration was observed previously in Pseudomonas aeruginosa, where the concentration of vitamin B12 ranged from 0.84 to 3.72, hence changed four-fold, depending on a strain ${ }^{28}$. 


\begin{tabular}{|c|c|c|c|c|c|c|c|c|c|}
\hline & Species & $\begin{array}{l}\text { M. tuberculosis } \\
\text { H37Rv }\end{array}$ & $\begin{array}{l}\text { M. abscessus subsp. } \\
\text { abscessus }\end{array}$ & $\begin{array}{l}\text { M. abscessus subsp. } \\
\text { bolletii }\end{array}$ & M. conspicuum & M. fortuitum & M. gastri & M. gordonae & M. innocens \\
\hline & Accession number & NC_000962 & NC_010397 & CP014950 & GCA_010730195 & CP011269 & LQOX1000000 & CP059165 & LS999933 \\
\hline & Strain & H37Rv & ATCC 19977 & FLAC 003 & JCM 14738 & CT6 & DSM 43505 & $24 \mathrm{~T}$ & MK13 \\
\hline & Life cycle & $\begin{array}{l}\text { Obligatory } \\
\text { pathogen }\end{array}$ & Opportunistic & Opportunistic & Opportunistic & Opportunistic & Opportunistic & Opportunistic & Opportunistic \\
\hline & Growth rate & Slow growing & Fast growing & Fast growing & Slow growing & Fast growing & Slow growing & Slow growing & Slow growing \\
\hline Gene name & \multicolumn{9}{|l|}{ Function } \\
\hline \multicolumn{10}{|c|}{ Aerobic pathway } \\
\hline - & \begin{tabular}{|l|} 
Precorrin-3B \\
methylase, predicted \\
replacement for $c o b F$
\end{tabular} & Rv2067c & & & & & & & \\
\hline - & $\begin{array}{l}\text { Bifunctional protein } \\
\text { Rnase } \mathrm{H} / \mathrm{cobC}\end{array}$ & Rv2228c & & & & & & & \\
\hline$c o b F$ & $\begin{array}{l}\text { Precorrin-6A } \\
\text { synthase }\end{array}$ & - & - & - & MCNS_43990 & - & AWC07_18155 & H0P51_RS23435 & EET03_RS22025 \\
\hline$c o b A$ & $\begin{array}{l}\text { Probable cob(I)ala- } \\
\text { min adenosyltrans- } \\
\text { ferase CobO }\end{array}$ & Rv2849c & - & - & MCNS_15910 & XA26_25280 & AWC07_16325 & H0P51_RS10520 & EET03_RS08695 \\
\hline$c o b B$ & $\begin{array}{l}\text { Cobyrinic acid A,C- } \\
\text { diamide synthase }\end{array}$ & Rv2848c & MAB_3155c & - & MCNS_15900 & - & AWC07_16320 & - & - \\
\hline$c o b C$ & \begin{tabular}{|l} 
L-threonine \\
3-O-phosphate \\
decarboxylase
\end{tabular} & Rv2231c & MAB_1902 & - & - & - & - & - & - \\
\hline$c o b D$ & $\begin{array}{l}\text { Adenosylcobina- } \\
\text { mide-phosphate } \\
\text { synthase }\end{array}$ & Rv2236c & MAB_1898 & - & MCNS_32370 & - & AWC07_23980 & - & - \\
\hline$c o b G$ & $\begin{array}{l}\text { Precorrin-3B } \\
\text { synthase }\end{array}$ & Rv2064 & MAB_2200c & - & MCNS_30550 & XA26_35740 & AWC07_17760 & H0P51_RS16405 & EET03_RS14985 \\
\hline$c o b H$ & $\begin{array}{l}\text { Cobalt-precor- } \\
\text { rin- } 8 \times \text { methylmutase }\end{array}$ & Rv2065 & MAB_2199c & - & MCNS_30560 & - & AWC07_17765 & - & - \\
\hline cobIJ & $\begin{array}{l}\text { Cobalt-precorrin-2 } \\
\text { C20-methyltrans- } \\
\text { ferase }\end{array}$ & Rv2066 & - & - & MCNS_30570 & - & AWC07_17770 & - & - \\
\hline$c o b K$ & $\begin{array}{l}\text { Cobalt-precor- } \\
\text { rin- } 6 \times \text { reductase }\end{array}$ & Rv2070c & MAB_2197 & - & MCNS_30570 & - & AWC07_17795 & - & - \\
\hline$c o b L$ & $\begin{array}{l}\text { Cobalt-precorrin-6y } \\
\text { C5-methyltrans- } \\
\text { ferase }\end{array}$ & Rv2072c & MAB_2195 & - & MCNS_30630 & - & AWC07_17805 & - & - \\
\hline$c o b M$ & $\begin{array}{l}\text { Cobalt-precorrin-4 } \\
\text { C11-methyltrans- } \\
\text { ferase }\end{array}$ & Rv2071c & MAB_2196 & - & MCNS_30620 & XA26_35820 & AWC07_17800 & H0P51_RS16430 & EET03_RS15035 \\
\hline$c o b N$ & Cobalt chelatase & Rv2062c & MAB_2201 & A3N95_10105 & MCNS_30500 & XA26_35720 & AWC07_17750 & H0P51_RS16365 & EET03_RS14960 \\
\hline$c o b O$ & $\begin{array}{l}\text { Cob(I)alamin adeno- } \\
\text { syltransferase }\end{array}$ & Rv2849c & MAB_3156c & - & MCNS_15890 & XA26_25260 & AWC07_16315 & H0P51_RS10510 & EET03_RS08685 \\
\hline $\operatorname{cobP}$ & $\begin{array}{l}\text { Adenosylcobinamide } \\
\text { kinase/adenosylco- } \\
\text { binamide phosphate } \\
\text { guanyltransferase }\end{array}$ & - & - & - & - & - & AWC07_21235 & - & - \\
\hline$c o b Q 1$ & $\begin{array}{l}\text { Cobyric acid } \\
\text { synthase }\end{array}$ & Rv0255c & - & A3N95_14850 & MCNS_15750 & - & - & - & - \\
\hline$c o b Q 2$ & \multirow{2}{*}{$\begin{array}{l}\text { Putative amidotrans- } \\
\text { ferase similar to } \\
\text { cobyric acid synthase }\end{array}$} & Rv3713 & MAB_0323c & - & MCNS_50840 & - & - & - & - \\
\hline$c o b R$ & & - & - & - & - & - & - & - & - \\
\hline cobs & Cobalamin synthase & Rv2208 & MAB_1952c & - & MCNS_31810 & - & AWC07_10855 & - & - \\
\hline $\operatorname{cob} T$ & $\begin{array}{l}\text { Nicotinate-nucleo- } \\
\text { tide-dimethylbenzi- } \\
\text { midazole phosphori- } \\
\text { bosyltransferase }\end{array}$ & Rv2207 & MAB_1953c & - & MCNS_31800 & XA26_37260 & AWC07_10860 & H0P51_RS17060 & EET03_RS15820 \\
\hline $\operatorname{cobU}$ & \multirow{2}{*}{$\begin{array}{l}\text { Adenosylcobina- } \\
\text { mide-phosphate } \\
\text { guanylyltransferase }\end{array}$} & Rv0254c & MAB_1954c & - & MCNS_31790 & - & AWC07_21235 & - & - \\
\hline $\operatorname{cobV}$ & & - & - & - & - & - & - & - & - \\
\hline$p d u O$ & \multirow{2}{*}{$\begin{array}{l}\mathrm{Cob}(\mathrm{I}) \text { alamin adeno- } \\
\text { syltransferase }\end{array}$} & Rv1314c & - & - & - & XA26_43420 & AWC07_13115 & - & - \\
\hline$p d u X$ & & - & - & - & - & - & - & - & - \\
\hline bluB & $\begin{array}{l}\text { 5,6-Dimethylbenzi- } \\
\text { midazole synthase }\end{array}$ & Rv0306 & - & - & MCNS_01070 & XA26_53750 & AWC07_19195 & H0P51_RS03095 & EET03_RS01545 \\
\hline \multicolumn{10}{|c|}{ Salvage pathway and transport } \\
\hline$b a c A$ & \multirow{2}{*}{$\begin{array}{l}\text { Cobalamin trans- } \\
\text { porter }\end{array}$} & Rv1819c & - & - & - & - & - & - & - \\
\hline$b t u B$ & & - & & - & - & - & - & - & - \\
\hline$b t u C$ & \multirow{3}{*}{$\begin{array}{l}\text { Iron } \mathrm{ABC} \text { trans- } \\
\text { porter permease }\end{array}$} & Rv2060 & - & - & - & - & - & - & - \\
\hline$b t u D$ & & - & - & - & - & - & - & - & - \\
\hline$b t u F$ & & - & - & - & - & - & - & - & - \\
\hline \multicolumn{10}{|c|}{ Anaerobic pathway } \\
\hline$c b i A$ & \multirow{2}{*}{$\begin{array}{l}\text { CbiA domain- } \\
\text { containing protein }\end{array}$} & - & - & - & - & - & - & - & - \\
\hline$c b i B$ & & - & - & - & - & - & - & - & - \\
\hline$c b i C$ & \multirow{2}{*}{$\begin{array}{l}\text { Precorrin-8X meth- } \\
\text { ylmutase }\end{array}$} & - & - & - & - & - & AWC07_17765 & - & - \\
\hline$c b i D$ & & - & - & - & - & - & - & - & - \\
\hline
\end{tabular}




\begin{tabular}{|c|c|c|c|c|c|c|c|c|c|}
\hline & Species & $\begin{array}{l}\text { M. tuberculosis } \\
\text { H37Rv }\end{array}$ & $\begin{array}{l}\text { M. abscessus subsp. } \\
\text { abscessus }\end{array}$ & $\begin{array}{l}\text { M. abscessus subsp. } \\
\text { bolletii }\end{array}$ & M. conspicuum & M. fortuitum & M. gastri & M. gordonae & M. innocens \\
\hline & Accession number & NC_000962 & NC_010397 & CP014950 & GCA_010730195 & CP011269 & LQOX1000000 & CP059165 & LS999933 \\
\hline & Strain & H37Rv & ATCC 19977 & FLAC 003 & JCM 14738 & CT6 & DSM 43505 & $24 \mathrm{~T}$ & MK13 \\
\hline & Life cycle & $\begin{array}{l}\text { Obligatory } \\
\text { pathogen }\end{array}$ & Opportunistic & Opportunistic & Opportunistic & Opportunistic & Opportunistic & Opportunistic & Opportunistic \\
\hline & Growth rate & Slow growing & Fast growing & Fast growing & Slow growing & Fast growing & \begin{tabular}{|l} 
Slow growing \\
\end{tabular} & \begin{tabular}{|l} 
Slow growing \\
\end{tabular} & Slow growing \\
\hline Gene name & \multicolumn{9}{|l|}{ Function } \\
\hline$c b i E$ & $\begin{array}{l}\text { Precorrin-6Y } \\
\text { C(5,15)-methyltrans- } \\
\text { ferase }\end{array}$ & - & - & - & MCNS_30630 & - & AWC07_17805 & H0P51_RS16435 & - \\
\hline$c b i F$ & \multirow{2}{*}{$\begin{array}{l}\text { Precorrin-4 C(11)- } \\
\text { methyltransferase }\end{array}$} & - & - & - & - & - & AWC07_17800 & - & - \\
\hline$c b i G$ & & - & - & - & - & - & - & - & - \\
\hline$c b i H$ & ATP-binding protein & - & - & - & - & - & AWC07_17770 & - & - \\
\hline$c b i J$ & \multirow{2}{*}{$\begin{array}{l}\text { Cobalt-precorrin-6A } \\
\text { reductase }\end{array}$} & - & - & - & - & - & AWC07_17795 & - & - \\
\hline$c b i K$ & & - & - & - & - & - & - & - & - \\
\hline$c b i L$ & \multirow{2}{*}{ ATP-binding protein } & - & - & - & - & - & AWC07_17770 & - & - \\
\hline$c b i P$ & & - & - & - & - & - & - & - & - \\
\hline$c b i T$ & $\begin{array}{l}\text { Precorrin-6Y-meth- } \\
\text { ylase }\end{array}$ & - & - & - & - & - & AWC07_17805 & - & - \\
\hline$c b i X$ & \begin{tabular}{|l|} 
Sirohydrochlorin \\
ferrochelatase
\end{tabular} & Rv0259c & - & A3N95_07805 & - & - & AWC07_14695 & - & - \\
\hline \multicolumn{10}{|c|}{ Urpoporfirynogen III pathway } \\
\hline$c y s G$ & \begin{tabular}{|l|} 
Multifunctional \\
uroporphyrin-III \\
C-methyltransferase/ \\
precorrin-2 oxidase/ \\
ferrochelatase
\end{tabular} & Rv2847c & MAB_3143c & - & MCNS_15910 & - & AWC07_16325 & - & - \\
\hline$c y s H$ & $\begin{array}{l}\text { Phosphoadenylyl- } \\
\text { sulfate reductase }\end{array}$ & Rv2392 & MAB_1661c & - & MCNS_35620 & - & AWC07_21315 & - & - \\
\hline$g l t X$ & $\begin{array}{l}\text { Glutamyl-tRNA } \\
\text { synthetase }\end{array}$ & Rv2992c & MAB_3298c & - & - & - & AWC07_23235 & - & - \\
\hline hemA & $\begin{array}{l}\text { Glutamyl-tRNA } \\
\text { reductase }\end{array}$ & Rv0509 & MAB_3993c & - & MCNS_03960 & - & AWC07_11800 & - & - \\
\hline hem $B$ & $\begin{array}{l}\text { Probable delta- } \\
\text { aminolevulinic acid } \\
\text { dehydratase/porpho- } \\
\text { bilinogen synthase }\end{array}$ & Rv0512 & MAB_3990c & - & MCNS_03990 & XA26_52000 & - & H0P51_RS04270 & EET03_RS02885 \\
\hline hem $C$ & $\begin{array}{l}\text { Porphobilinogen } \\
\text { deaminase }\end{array}$ & Rv0510 & MAB_3992c & - & MCNS_03970 & XA26_52020 & AWC07_11795 & H0P51_RS04260 & EET03_RS02875 \\
\hline hemD & $\begin{array}{l}\text { Uroporphyrinogen } \\
\text { III methyltrans- } \\
\text { ferase/synthase }\end{array}$ & Rv0511 & - & - & MCNS_03980 & - & AWC07_14690 & - & - \\
\hline hemL & \begin{tabular}{|l} 
Glutamate- \\
1-semialdehyde \\
2,1-aminomutase
\end{tabular} & Rv0524 & - & - & MCNS_04110 & XA26_51830 & AWC07_11675 & H0P51_RS04340 & EET03_RS03025 \\
\hline $\operatorname{hem} Y$ & $\begin{array}{l}\text { Chll component of } \\
\text { cobalt chelatase }\end{array}$ & Rv2850c & MAB_2985c & - & MCNS_17540 & - & - & - & - \\
\hline \multicolumn{10}{|c|}{ Vitamin B12 dependent enzymes } \\
\hline metH & $\begin{array}{l}\text { 5-Methyltetrahydro- } \\
\text { folate-homocysteine } \\
\text { methyltransferase }\end{array}$ & Rv2124c & MAB_2129 & - & MCNS_30990 & - & AWC07_11205 & H0P51_RS04340 & EET03_RS15385 \\
\hline$m u t B$ & $\begin{array}{l}\text { Methylmalonyl-CoA } \\
\text { mutase }\end{array}$ & Rv1493 & MAB_2711c & - & MCNS_22010 & - & - & - & - \\
\hline \multirow[t]{6}{*}{$n r d Z$} & $\begin{array}{l}\text { Ribonucleotide } \\
\text { reductase of class II }\end{array}$ & Rv0570 & - & - & - & - & AWC07_08365 & - & - \\
\hline & Species & M. kansasii & M. persicum & M. phlei & M. porcinum & M. terrae & M. xenopi & M.szulgai & M. smegmatis \\
\hline & Accession number & GCA_000157895.1 & GCA_002705835 & GCA_001582015 & $\begin{array}{l}\text { NZ } \\
\text { MBDY01000007.1 }\end{array}$ & GCA_900187145 & $\begin{array}{l}\text { NZ_- } \\
\text { AJFI01000095.1 }\end{array}$ & NZ_LQPW01000016.1 & СР000480 \\
\hline & Strain & ATCC 12478 & $\mathrm{H} 48$ & CCUG 21000 & ACS 3670 & NCTC 10856 & RIVM700366 & DSM 44166 & mc2 155 \\
\hline & Life cycle & Opportunistic & Opportunistic & Opportunistic & Opportunistic & Opportunistic & Opportunistic & Pathogenic & Non-pathogenic \\
\hline & Growth rate & Slow growing & Slow growing & Fast growing & Fast growing & Slow growing & Slow growing & Slow growing & Fast growing \\
\hline \multicolumn{10}{|c|}{ Aerobic pathway } \\
\hline- & $\begin{array}{l}\text { Precorrin-3B } \\
\text { methylase, predicted } \\
\text { replacement for } \\
\text { cobF }\end{array}$ & & & & & & & & \\
\hline- & $\begin{array}{l}\text { Bifunctional protein } \\
\text { Rnase H/cobC }\end{array}$ & & & & & & & & \\
\hline$c o b F$ & $\begin{array}{l}\text { Precorrin-6A } \\
\text { synthase }\end{array}$ & MKAN_RS08645 & - & - & A5717_31225 & - & MXEN_19174 & - & MSMEG_5548 \\
\hline $\operatorname{cob} A$ & $\begin{array}{l}\text { Probable cob(I)ala- } \\
\text { min adenosyltrans- } \\
\text { ferase CobO }\end{array}$ & MKAN_RS09965 & CDN37_RS24000 & - & A5717_05685 & - & MXEN_03569 & AWC27_RS04140 & \\
\hline$c o b B$ & $\begin{array}{l}\begin{array}{l}\text { Cobyrinic acid A,C- } \\
\text { diamide synthase }\end{array} \\
\end{array}$ & - & - & - & A5717_05680 & - & MXEN_03564 & - & MSMEG_2617 \\
\hline$c o b C$ & $\begin{array}{l}\text { L-threonine } \\
\text { 3-O-phosphate } \\
\text { decarboxylase }\end{array}$ & - & - & - & - & - & - & - & - \\
\hline \multicolumn{10}{|l|}{ Continued } \\
\hline
\end{tabular}




\begin{tabular}{|c|c|c|c|c|c|c|c|c|c|}
\hline$c o b D$ & $\begin{array}{l}\text { Adenosylcobina- } \\
\text { mide-phosphate } \\
\text { synthase }\end{array}$ & MKAN_03275 & - & - & A5717_01680 & - & MXEN_00720 & - & MSMEG_4310 \\
\hline$c o b G$ & $\begin{array}{l}\text { Precorrin-3B } \\
\text { synthase }\end{array}$ & MKAN_RS01775 & CDN37_RS01855 & $\begin{array}{l}\text { MPHLCCUG_ } \\
\text { RS13200 }\end{array}$ & A5717_31635 & - & MXEN_01317 & AWC27_RS14575 & MSMEG_3871 \\
\hline $\mathrm{cobH}$ & $\begin{array}{l}\text { Cobalt-precor- } \\
\text { rin- } 8 \times \text { methyl- } \\
\text { mutase }\end{array}$ & - & - & - & A5717_31640 & - & MXEN_01322 & - & MSMEG_3872 \\
\hline cobIJ & $\begin{array}{l}\text { Cobalt-precorrin-2 } \\
\text { C20-methyltrans- } \\
\text { ferase }\end{array}$ & - & - & - & A5717_31645 & - & MXEN_01327 & - & MSMEG_3873 \\
\hline$c o b K$ & $\begin{array}{l}\text { Cobalt-precor- } \\
\text { rin-6 } 6 \text { reductase }\end{array}$ & - & - & - & A5717_31665 & - & MXEN_01342 & - & MSMEG_3875 \\
\hline$c o b L$ & $\begin{array}{l}\text { Cobalt-precorrin-6y } \\
\text { C5-methyltrans- } \\
\text { ferase }\end{array}$ & - & - & - & A5717_31675 & - & MXEN_01352 & - & MSMEG_3878 \\
\hline $\operatorname{cobM}$ & $\begin{array}{l}\text { Cobalt-precorrin-4 } \\
\text { C11-methyltrans- } \\
\text { ferase }\end{array}$ & MKAN_RS01815 & CDN37_RS01895 & $\begin{array}{l}\text { MPHLCCUG_ } \\
\text { RS13225 }\end{array}$ & A5717_31670 & - & MXEN_01347 & - & MSMEG_3877 \\
\hline$c o b N$ & Cobalt chelatase & MKAN_RS01760 & CDN37_RS01840 & $\begin{array}{l}\text { MPHLCCUG_ } \\
\text { RS12530 }\end{array}$ & A5717_31630 & - & MXEN_01292 & AWC27_RS14585 & MSMEG_3864 \\
\hline$c o b O$ & \begin{tabular}{|l|}
$\mathrm{Cob}(\mathrm{I})$ alamin \\
adenosyltransferase
\end{tabular} & MKAN_RS23620 & CDN37_RS23990 & $\begin{array}{l}\text { MPHLCCUG_ } \\
\text { RS15725 }\end{array}$ & A5717_05675 & - & MXEN_03569 & AWC27_RS04130 & MSMEG_2616 \\
\hline$c o b P$ & $\begin{array}{l}\text { Adenosyl- } \\
\text { cobinamide kinase/ } \\
\text { adenosylcobinamide } \\
\text { phosphate guanyl- } \\
\text { transferase }\end{array}$ & - & - & - & A5717_01465 & - & MXEN_00460 & - & - \\
\hline$c o b Q 1$ & $\begin{array}{l}\text { Cobyric acid } \\
\text { synthase }\end{array}$ & - & - & - & - & - & - & - & MSMEG_2588 \\
\hline$c o b Q 2$ & \multirow{2}{*}{$\begin{array}{l}\text { Putative ami- } \\
\text { dotransferase } \\
\text { similar to cobyric } \\
\text { acid synthase }\end{array}$} & - & - & - & - & - & MXEN_13996 & - & - \\
\hline$c o b R$ & & - & - & - & - & - & - & - & - \\
\hline cobs & Cobalamin synthase & - & - & - & A5717_01475 & SAMEA4434518_01622 & MXEN_00470 & - & MSMEG_4277 \\
\hline$c o b T$ & $\begin{array}{l}\text { Nicotinate- } \\
\text { nucleotide-dimeth- } \\
\text { ylbenzimidazole } \\
\text { phosphoribosyl- } \\
\text { transferase }\end{array}$ & MKAN_RS02865 & CDN37_RS02735 & $\begin{array}{l}\text { MPHLCCUG_ } \\
\text { RS16365 }\end{array}$ & A5717_01470 & SAMEA4434518_01623 & MXEN_00465 & AWC27_RS19945 & MSMEG_4275 \\
\hline$c o b U$ & \multirow{2}{*}{$\begin{array}{l}\text { Adenosylcobina- } \\
\text { mide-phosphate } \\
\text { guanylyltransferase }\end{array}$} & - & - & - & A5717_01465 & SAMEA4434518_00431 & MXEN_00460 & - & MSMEG_4274 \\
\hline $\operatorname{cobV}$ & & - & - & - & - & - & - & - & - \\
\hline$p d u O$ & \multirow{2}{*}{$\begin{array}{l}\mathrm{Cob}(\mathrm{I}) \text { alamin } \\
\text { adenosyltransferase }\end{array}$} & - & - & - & A5717_18045 & - & MXEN_16843 & - & MSMEG_1544 \\
\hline$p d u X$ & & - & - & - & - & - & - & - & - \\
\hline bluB & $\begin{array}{l}\text { 5,6-Dimethylbenzi- } \\
\text { midazole synthase }\end{array}$ & MKAN_RS16250 & CDN37_RS16575 & $\begin{array}{l}\text { MPHLCCUG_ } \\
\text { RS02360 }\end{array}$ & A5717_14585 & - & MXEN_19875 & AWC27_RS20945 & MSMEG_6053 \\
\hline \multicolumn{10}{|c|}{ Salvage pathway and transport } \\
\hline bacA & \multirow{2}{*}{$\begin{array}{l}\text { Cobalamin trans- } \\
\text { porter }\end{array}$} & - & - & - & - & - & - & - & - \\
\hline$b t u B$ & & - & - & - & - & - & - & - & - \\
\hline btuc & \multirow{3}{*}{$\begin{array}{l}\text { Iron } A B C \text { trans- } \\
\text { porter permease }\end{array}$} & - & - & - & A5717_14615 & - & MXEN_06686 & - & - \\
\hline$b t u D$ & & - & - & - & - & - & - & - & - \\
\hline$b t u F$ & & - & - & - & - & - & - & - & - \\
\hline \multicolumn{10}{|c|}{ Anaerobic pathway } \\
\hline$c b i A$ & \multirow{2}{*}{$\begin{array}{l}\text { CbiA domain- } \\
\text { containing protein }\end{array}$} & - & - & - & - & - & MXEN_04563 & - & - \\
\hline$c b i B$ & & - & - & - & - & - & - & - & - \\
\hline$c b i C$ & \multirow{2}{*}{$\begin{array}{l}\text { Precorrin-8X } \\
\text { methylmutase }\end{array}$} & - & - & - & A5717_31640 & - & - & - & - \\
\hline$c b i D$ & & - & - & - & - & - & - & - & - \\
\hline$c b i E$ & $\begin{array}{l}\text { Precorrin-6Y } \\
\mathrm{C}(5,15) \text {-methyl- } \\
\text { transferase }\end{array}$ & - & CDN37_RS01900 & - & A5717_31675 & - & MXEN_01352 & AWC27_RS14545 & - \\
\hline$c b i F$ & \multirow{2}{*}{$\begin{array}{l}\text { Precorrin-4 C(11)- } \\
\text { methyltransferase }\end{array}$} & - & - & - & A5717_31670 & - & MXEN_01347 & - & - \\
\hline$c b i G$ & & - & - & - & - & - & - & - & - \\
\hline$c b i H$ & $\begin{array}{l}\text { ATP-binding } \\
\text { protein }\end{array}$ & - & - & - & A5717_31645 & - & - & - & - \\
\hline$c b i J$ & \multirow{2}{*}{$\begin{array}{l}\text { Cobalt-precorrin- } \\
6 \mathrm{~A} \text { reductase }\end{array}$} & - & - & - & A5717_31665 & - & MXEN_01342 & - & - \\
\hline$c b i K$ & & - & - & - & - & - & - & - & - \\
\hline$c b i L$ & \multirow{2}{*}{$\begin{array}{l}\text { ATP-binding } \\
\text { protein }\end{array}$} & - & - & - & A5717_31645 & - & - & - & - \\
\hline$c b i P$ & & - & - & - & - & - & - & - & - \\
\hline$c b i T$ & $\begin{array}{l}\text { Precorrin-6Y-meth- } \\
\text { ylase }\end{array}$ & - & - & - & A5717_31675 & - & MXEN_01352 & - & - \\
\hline$c b i X$ & $\begin{array}{l}\text { Sirohydrochlorin } \\
\text { ferrochelatase }\end{array}$ & - & - & - & A5717_10190 & SAMEA4434518_00227 & MXEN_11286 & - & - \\
\hline \multicolumn{10}{|c|}{ Urpoporfirynogen III pathway } \\
\hline$c y s G$ & \begin{tabular}{|l|} 
Multifunctional \\
uroporphyrin-III \\
C-methyltrans- \\
ferase/precorrin-2 \\
oxidase/ferroche- \\
latase
\end{tabular} & - & - & - & A5717_05685 & - & MXEN_03559 & - & - \\
\hline Contir & & & & & & & & & \\
\hline
\end{tabular}




\begin{tabular}{|c|c|c|c|c|c|c|c|c|c|}
\hline$c y s \mathrm{H}$ & $\begin{array}{l}\text { Phosphoadenylyl- } \\
\text { sulfate reductase }\end{array}$ & - & - & - & A5717_28590 & SAMEA4434518_01414 & MXEN_11291 & - & - \\
\hline$g l t X$ & $\begin{array}{l}\text { Glutamyl-tRNA } \\
\text { synthetase }\end{array}$ & - & - & - & A5717_14355 & - & MXEN_16257 & - & MSMEG_2383 \\
\hline hemA & $\begin{array}{l}\text { Glutamyl-tRNA } \\
\text { reductase }\end{array}$ & - & - & - & A5717_22190 & SAMEA4434518_00496 & MXEN_04673 & - & MSMEG_0919 \\
\hline hemB & $\begin{array}{l}\text { Probable delta- } \\
\text { aminolevulinic } \\
\text { acid dehydratase/ } \\
\text { porphobilinogen } \\
\text { synthase }\end{array}$ & MKAN_RS17655 & CDN37_RS17885 & $\begin{array}{l}\text { MPHLCCUG } \\
\text { RS22000 }\end{array}$ & - & SAMEA4434518_00499 & - & AWC27_RS21685 & MSMEG_0956 \\
\hline hem $C$ & $\begin{array}{l}\text { Porphobilinogen } \\
\text { deaminase }\end{array}$ & MKAN_RS17645 & CDN37_RS17875 & $\begin{array}{l}\text { MPHLCCUG } \\
\text { RS22010 }\end{array}$ & A5717_22195 & SAMEA4434518_00497 & MXEN_04668 & AWC27_RS07580 & MSMEG_0953 \\
\hline hemD & $\begin{array}{l}\text { Uroporphyrinogen } \\
\text { III methyltrans- } \\
\text { ferase/synthase }\end{array}$ & - & - & - & A5717_10195 & SAMEA4434518_00498 & MXEN_04663 & - & MSMEG_0954 \\
\hline hemL & $\begin{array}{l}\text { Glutamate-1-semi- } \\
\text { aldehyde 2,1-amino- } \\
\text { mutase }\end{array}$ & MKAN_RS17800 & CDN37_RS18005 & $\begin{array}{l}\text { MPHLCCUG } \\
\text { RS21920 }\end{array}$ & A5717_22280 & SAMEA4434518_00514 & MXEN_04593 & AWC27_RS07655 & MSMEG_0969 \\
\hline hem $Y$ & $\begin{array}{l}\text { Chll component of } \\
\text { cobalt chelatase }\end{array}$ & - & - & - & - & SAMEA4434518_01694 & - & - & - \\
\hline \multicolumn{10}{|c|}{ Vitamin B12 dependent enzymes } \\
\hline$m e t H$ & $\begin{array}{l}\text { 5-Methyltetrahydro- } \\
\text { folate-homocyst- } \\
\text { eine methyltrans- } \\
\text { ferase }\end{array}$ & - & CDN37_RS02225 & $\begin{array}{l}\text { MPHLCCUG } \\
\text { RS15920 }\end{array}$ & A5717_31970 & SAMEA4434518_01721 & MXEN_01507 & AWC27_RS09650 & MSMEG_0093 \\
\hline mutB & $\begin{array}{l}\text { Methylmalonyl- } \\
\text { CoA mutase }\end{array}$ & - & - & - & - & SAMEA4434518_02142 & - & - & MSMEG_3159 \\
\hline$n r d Z$ & $\begin{array}{l}\text { Ribonucleotide } \\
\text { reductase of class II }\end{array}$ & MKAN_19005 & - & - & - & - & MXEN_17528 & - & - \\
\hline
\end{tabular}

Table 1. Identification of genes involved in vitamin B12 metabolism in various mycobacteria species based on publicly available annotations in major databases.

Possible sources of the variability in the production of vitamin B12 in different strains are mutations either in the promoter regions of genes involved in the synthesis or directly in coding sequences, resulting in enzymes with altered reaction rates ${ }^{30}$.

Strain variability in the level of vitamin B12 production is important in the context of M. tuberculosis. Data presented in previous manuscripts suggested indirectly that certain strains of $M$. tuberculosis may be capable of cobalamin synthesis, while others are probably not ${ }^{14,31}$. As in other species, mycobacteria do show a certain spread in the level of vitamin B12 synthesis that probably can be attributed to the genetic background rather than the environmental factors or stage of the growth.

Increased concentration of vitamin B12 in mycobacterial cells under starvation results from accumulation rather than increased production. In our previous study, we showed that cells of $M$. smegmatis grown in a medium deprived of nutrients contain an approximately eightfold amount of vitamin B12 when compared to cultures grown in a rich broth. An increase in vitamin B12 concentration was also observed in stationary phase cultures ${ }^{17}$. A similar observation was made in $P$. aeruginosa. There, vitamin B12 concentration increased from non-detectable during exponential growth to $0.32-0.67 \mathrm{ng} / \mathrm{mg}$ of protein in stationary phase cultures, depending on a strain. The concentration further increased up to 3.72 in conditions of continuous-flow growth ${ }^{28}$.

Here, we show that the reason behind the increased concentration of vitamin B12 in starved cells of M. smegmatis $\mathrm{mc}^{2}$ probably results from accumulation rather than increased synthesis. We estimated the relative gene expression of genes involved in cobalamin synthesis in starved cells compared to cells in the logarithmic phase (Fig. 4). We observed that the expression of genes involved in vitamin B12 synthesis was either constitutive (0 to 1 -fold change in relative expression to sigA) for $c o b G, \operatorname{cobL}$, cobO and $c o b D$ or repressed (>3-fold change) for $c o b U$ and $c o b N$.

Accumulation of vitamin B12 in starved cells and old cultures of M. smegmatis is important in the context of cobalamin detection in M. tuberculosis. M. smegmatis is a model organism for studying the biology of mycobacteria, including M. tuberculosis ${ }^{32}$. Bacteria of the same phylogenetic order are likely to maintain the same biological pathways and mechanisms. Indeed, increased expression of cobalamin synthesis genes was reported in dormant cultures of $M$. tuberculosis ${ }^{16}$. Therefore, if cobalamin was to be present in the cells of M. tuberculosis, it was more likely to be identified in prolonged, starved, or dormant cultures.

Lack of observable vitamin B12 production in M. tuberculosis. We tested the contents of cells of $M$. tuberculosis for vitamin B12 by immunoassay (Table 3). We included laboratory strain of $M$. tuberculosis $\mathrm{H} 37 \mathrm{Rv}$ and five clinical strains of $M$. tuberculosis, here grouped into a group of "clinical strains". As a negative control strain, we used M. tuberculosis deficient in cobIJ gene. Predicted function of cobIJ is precorrin-2 C20-methyltransferase/precorrin-3B C17-methyltransferase. The gene product is required at the early stage of vitamin B12 synthesis (Fig. 1).

We screened M. tuberculosis cell lysates derived from cultures grown in various conditions. The growth conditions aimed to mimic the environments that can be found during M. tuberculosis infection cycle. All cultures were 


\begin{tabular}{|c|c|c|c|c|c|c|c|c|}
\hline \multirow[b]{2}{*}{ Gene name } & \multirow[b]{2}{*}{ Description } & \multicolumn{3}{|c|}{ M. abscessus subsp. abscessus } & \multicolumn{3}{|l|}{ M. smegmatis } & \\
\hline & & Locus & Average & SD & Locus & Average & SD & \\
\hline All genes & & & 201.88 & 547.4 & & 147.33 & 607.04 & \\
\hline \multicolumn{9}{|c|}{ Reference genes } \\
\hline sigA & RNA polymerase sigma factor SigA (sigma-A) & MAB_3009 & 1213.5 & 31.5941962 & - & - & - & \\
\hline dnaA & Chromosomal replication initiator protein DnaA & MAB_0001 & 285.34 & 4.39631664 & MSMEG_0093 & 94.66 & 39.15167557 & \\
\hline $\mathrm{ftsZ}$ & Cell division protein FtsZ & MAB_2009 & 624.6633333 & 540.8220485 & MSMEG_4222 & 299.37 & 47.21376812 & \\
\hline dnaG & Probable DNA primase DnaG & MAB_1708 & 154.3033333 & 9.405750014 & - & - & - & \\
\hline rpoB & $\begin{array}{l}\text { DNA-directed RNA polymerase (beta chain) RpoB (tran- } \\
\text { scriptase beta chain) (RNA polymerase beta subunit) }\end{array}$ & MAB_3869c & 2151.213333 & 32.03047351 & MSMEG_1367 & 511.1433333 & 441.0141973 & \\
\hline \multicolumn{9}{|c|}{ Aerobic pathway } \\
\hline- & Precorrin-3B methylase, predicted replacement for cobF & - & - & - & - & - & - & \\
\hline- & Bifunctional protein Rnase $\mathrm{H} / \mathrm{cobC}$ & - & - & - & - & - & - & \\
\hline $\operatorname{cobA}$ & Probable cob(I)alamin adenosyltransferase CobO & - & - & - & - & - & - & \\
\hline $\operatorname{cobB}$ & Cobyrinic acid A,C-diamide synthase & MAB_3155c & 48.28333333 & 3.208899084 & MSMEG_2617 & 45.33666667 & 37.90995428 & \\
\hline $\operatorname{cobC}$ & L-threonine 3-O-phosphate decarboxylase & MAB_1902 & 35.61333333 & 2.020948622 & - & - & - & \\
\hline cobD & Adenosylcobinamide-phosphate synthase & MAB_1898 & 30.22333333 & 2.78363671 & MSMEG_4310 & 34.71333333 & 5.899494329 & \\
\hline $\operatorname{cobG}$ & Precorrin-3B synthase & MAB_2200c & 44.81333333 & 10.51069138 & MSMEG_3871 & 43.05333333 & 9.251671921 & \\
\hline cobH & Cobalt-precorrin $-8 \times$ methylmutase & MAB_2199c & 53.07 & 1.728670009 & MSMEG_3872 & 110.2366667 & 37.68649802 & \\
\hline cobIJ & Cobalt-precorrin-2 C20-methyltransferase & - & - & - & MSMEG_3873 & 52.53333333 & 35.62759651 & \\
\hline cobK & Cobalt-precorrin- $6 \times$ reductase & MAB_2197 & 118.63 & 8.898027871 & MSMEG_3875 & 40.99 & 6.827935266 & \\
\hline cobL & Cobalt-precorrin-6y C5-methyltransferase & MAB_2195 & 95.33 & 10.65838168 & MSMEG_3878 & 33.24333333 & 4.282117856 & \\
\hline $\operatorname{cobM}$ & Cobalt-precorrin-4 C11-methyltransferase & MAB_2196 & 190.0066667 & 34.70278423 & MSMEG_3877 & 40.97333333 & 4.718308313 & \\
\hline $\operatorname{cobN}$ & Cobalt chelatase & MAB_2201 & 78.53 & 3.401043957 & MSMEG_3864 & 95.39333333 & 66.90839808 & \\
\hline cobO & $\mathrm{Cob}(\mathrm{I})$ alamin adenosyltransferase & MAB_3156c & 94.59 & 20.00465696 & MSMEG_2616 & 150.34 & 26.29276136 & \\
\hline cobQ1 & Cobyric acid synthase & - & - & - & MSMEG_2588 & 69.94666667 & 36.76729162 & \\
\hline cobQ2 & Putative amidotransferase similar to cobyric acid synthase & MAB_0323c & 79.06333333 & 7.416537827 & - & - & - & \\
\hline cobS & Cobalamin synthase & MAB_1952c & 98.49666667 & 3.519038694 & MSMEG_4277 & 35.19666667 & 3.883160225 & \\
\hline $\operatorname{cobT}$ & $\begin{array}{l}\text { Nicotinate-nucleotide-dimethylbenzimidazole phosphori- } \\
\text { bosyltransferase }\end{array}$ & MAB_1953c & 171.1133333 & 8.786639479 & MSMEG_4275 & 34.59333333 & 4.231930214 & \\
\hline cobU & Adenosylcobinamide-phosphate guanylyltransferase & MAB_1954c & 191.44 & 15.12232456 & MSMEG_4274 & 109.1266667 & 5.803363967 & \\
\hline pduO & $\operatorname{Cob}(\mathrm{I})$ alamin adenosyltransferase & - & - & - & MSMEG_1544 & 233.6766667 & 151.377732 & \\
\hline bluB & 5,6-Dimethylbenzimidazole synthase & - & - & - & MSMEG_6053 & 97.35333333 & 36.85650056 & \\
\hline \multicolumn{9}{|c|}{ Salvage pathway and transport } \\
\hline bacA & Cobalamin transporter & - & - & - & - & - & - & \\
\hline btuC & Iron $\mathrm{ABC}$ transporter permease & - & - & - & - & - & - & \\
\hline \multicolumn{9}{|c|}{ Anaerobic pathway } \\
\hline cbiX & Sirohydrochlorin ferrochelatase & - & - & - & - & - & - & \\
\hline \multicolumn{9}{|c|}{ Urpoporfirynogen III pathway } \\
\hline cysG & $\begin{array}{l}\text { Multifunctional uroporphyrin-III C-methyltransferase/ } \\
\text { precorrin-2 oxidase/ferrochelatase }\end{array}$ & MAB_3143c & 95.06666667 & 7.895709806 & - & - & - & \\
\hline cysH & Phosphoadenylyl-sulfate reductase & MAB_1661c & 174.4733333 & 18.36925783 & - & - & - & \\
\hline gltX & Glutamyl-tRNA synthetase & MAB_3298c & 287.6433333 & 11.14623853 & MSMEG_2383 & 156.9933333 & 19.13254383 & \\
\hline hemA & Glutamyl-tRNA reductase & MAB_3993c & 268.16 & 8.943438936 & MSMEG_0919 & 174.06 & 98.84548143 & \\
\hline hemB & $\begin{array}{l}\text { Probable delta-aminolevulinic acid dehydratase/porpho- } \\
\text { bilinogen synthase }\end{array}$ & MAB_3990c & 349.51 & 54.2694122 & MSMEG_0956 & 201.9733333 & 27.76189895 & \\
\hline hemC & Porphobilinogen deaminase & MAB_3992c & 638.4233333 & 82.95156076 & MSMEG_0953 & 374.95 & 313.6007022 & \\
\hline hemD & Uroporphyrinogen III methyltransferase/synthase & - & - & - & MSMEG_0954 & 317.5766667 & 44.41745753 & \\
\hline hemL & Glutamate-1-semialdehyde 2,1-aminomutase & - & - & - & MSMEG_0969 & 110.7066667 & 25.2243619 & \\
\hline hemY & ChlI component of cobalt chelatase & MAB_2985c & 196.3366667 & 1.652099674 & - & - & - & \\
\hline \multicolumn{9}{|c|}{ Vitamin B12 dependent enzymes } \\
\hline metH & 5-Methyltetrahydrofolate-homocysteine methyltransferase & MAB_2129 & 605.9966667 & 39.45958101 & MSMEG_0093 & 94.66 & 39.15167557 & \\
\hline mutB & Methylmalonyl-CoA mutase & MAB_2711c & 180.9466667 & 2.206928484 & - & - & - & \\
\hline $\operatorname{nrdZ}$ & Ribonucleotide reductase of class II & - & - & - & - & - & - & \\
\hline \multirow[b]{3}{*}{ Gene name } & \multirow[b]{3}{*}{ Description } & \multicolumn{7}{|c|}{ M. tuberculosis } \\
\hline & & \multirow[b]{2}{*}{ Locus } & \multicolumn{2}{|l|}{ Rich 7H9 broth } & Cholesterol & & Macrophages & \\
\hline & & & Average & SD & Average & SD & Average & SD \\
\hline All genes & & & 256.0163876 & 551.1120531 & 256.01 & 764.53 & 256.0163722 & 1039.716894 \\
\hline Reference ge & & & & & & & & \\
\hline sigA & RNA polymerase sigma factor SigA (sigma-A) & Rv2703 & 599.0566667 & 9.565264706 & 630.73 & 106.0339119 & 1776.92 & 403.8168363 \\
\hline $\operatorname{dnaA}$ & Chromosomal replication initiator protein DnaA & Rv0001 & 131.0233333 & 1.802615384 & 532.06 & 40.00305738 & 579.74 & 351.8220045 \\
\hline $\mathrm{ftsZ}$ & Cell division protein FtsZ & Rv2150c & 691.0366667 & 72.64343069 & 200.9733333 & 88.35943501 & 548.8 & 187.4937259 \\
\hline dnaG & Probable DNA primase DnaG & Rv2343c & 104.9866667 & 2.321354968 & 91.05666667 & 42.02332077 & 118.2366667 & 98.34518335 \\
\hline Continued & & & & & & & & \\
\hline
\end{tabular}




\begin{tabular}{|c|c|c|c|c|c|c|c|c|}
\hline \multirow[b]{3}{*}{ Gene name } & \multirow[b]{3}{*}{ Description } & \multicolumn{7}{|c|}{ M. tuberculosis } \\
\hline & & \multirow[b]{2}{*}{ Locus } & \multicolumn{2}{|l|}{ Rich $7 \mathrm{H} 9$ broth } & \multicolumn{2}{|l|}{ Cholesterol } & \multicolumn{2}{|l|}{ Macrophages } \\
\hline & & & Average & SD & Average & SD & Average & SD \\
\hline rpoB & $\begin{array}{l}\text { DNA-directed RNA polymerase (beta chain) RpoB (tran- } \\
\text { scriptase beta chain) (RNA polymerase beta subunit) }\end{array}$ & Rv0667 & 1650.64 & 35.26432853 & 701.45 & 81.97233192 & 438.5966667 & 130.3168532 \\
\hline \multicolumn{9}{|c|}{ Aerobic pathway } \\
\hline- & Precorrin-3B methylase, predicted replacement for cobF & Rv2067c & 104.4866667 & 4.4877339 & 32.906666667 & 33.05350242 & 283.67 & 60.07031935 \\
\hline- & Bifunctional protein Rnase $\mathrm{H} / \mathrm{cobC}$ & Rv2228c & 91.63333333 & 0.241430919 & 36.25333333 & 20.75320752 & 0 & 0 \\
\hline cobA & Probable cob(I)alamin adenosyltransferase CobO & Rv2849c & 77.32666667 & 3.099519676 & 74.58333333 & 10.26311302 & 507.9333333 & 458.6724143 \\
\hline $\operatorname{cobB}$ & Cobyrinic acid A,C-diamide synthase & Rv2848c & 51.04333333 & 0.651783877 & 60.56666667 & 31.54769652 & 0 & 0 \\
\hline cobC & L-threonine 3-O-phosphate decarboxylase & Rv2231c & 78.29 & 3.854279007 & 37.80333333 & 27.56415224 & 0 & 0 \\
\hline $\operatorname{cobD}$ & Adenosylcobinamide-phosphate synthase & Rv2236c & 58.52333333 & 1.689108904 & 1.9 & 1.064831755 & 81.79333333 & 115.6732413 \\
\hline cobG & Precorrin-3B synthase & Rv2064 & 183.29 & 4.164956983 & 33.45 & 26.14527236 & 133.5466667 & 188.8635072 \\
\hline cobH & Cobalt-precorrin $-8 \times$ methylmutase & Rv2065 & 178.23 & 4.38254112 & 0.593333333 & 0.839100047 & 0 & 0 \\
\hline cobIJ & Cobalt-precorrin-2 C20-methyltransferase & Rv2066 & 150.18 & 9.995749096 & 74.32666667 & 8.916786915 & 72.98 & 58.51295412 \\
\hline $\operatorname{cobK}$ & Cobalt-precorrin- $6 \times$ reductase & Rv2070c & 89.46666667 & 8.680419857 & 42.11333333 & 30.69800681 & 419.98 & 124.7825583 \\
\hline $\operatorname{cobL}$ & Cobalt-precorrin-6y C5-methyltransferase & Rv2072c & 51.73666667 & 2.388616522 & 56.36333333 & 44.1996458 & 219.44 & 310.3350241 \\
\hline $\operatorname{cobM}$ & Cobalt-precorrin-4 C11-methyltransferase & Rv2071c & 32.80333333 & 1.224100577 & 63.166666667 & 39.84209192 & 0 & 0 \\
\hline $\operatorname{cobN}$ & Cobalt chelatase & Rv2062c & 101.4866667 & 3.59177146 & 46.24666667 & 13.10098554 & 167.3266667 & 118.4526528 \\
\hline cobO & Cob(I)alamin adenosyltransferase & Rv2849c & 77.32666667 & 3.099519676 & 74.58333333 & 10.26311302 & 507.9333333 & 458.6724143 \\
\hline cobQ1 & Cobyric acid synthase & Rv0255c & 83.46333333 & 4.16039528 & 60.76333333 & 17.12575124 & 0 & 0 \\
\hline cobQ2 & Putative amidotransferase similar to cobyric acid synthase & Rv3713 & 178.4833333 & 3.066206487 & 95.84333333 & 45.96082849 & 104.7666667 & 148.1624409 \\
\hline $\operatorname{cobS}$ & Cobalamin synthase & Rv2208 & 280.7333333 & 5.75070044 & 38.15 & 35.52983347 & 228.8033333 & 323.5767771 \\
\hline $\operatorname{cobT}$ & $\begin{array}{l}\text { Nicotinate-nucleotide-dimethylbenzimidazole phosphori- } \\
\text { bosyltransferase }\end{array}$ & Rv2207 & 341.1866667 & 6.934096112 & 10.67 & 6.638649461 & 70.94666667 & 100.3337382 \\
\hline $\operatorname{cobU}$ & Adenosylcobinamide-phosphate guanylyltransferase & Rv0254c & 46.93666667 & 5.12625486 & 171.9133333 & 89.37495001 & 138.89 & 196.4201217 \\
\hline pduO & $\mathrm{Cob}(\mathrm{I})$ alamin adenosyltransferase & Rv1314c & 88.84 & 1.75789647 & 51.02 & 33.75337119 & 125.2866667 & 177.1821032 \\
\hline bluB & 5,6-Dimethylbenzimidazole synthase & Rv0306 & 50.93 & 3.946044433 & 74.76 & 42.37912772 & 0 & 0 \\
\hline \multicolumn{9}{|c|}{ Salvage pathway and transport } \\
\hline bacA & Cobalamin transporter & Rv1819c & 112.5766667 & 3.598373089 & 27.63 & 21.66365312 & 82.66333333 & 59.02668455 \\
\hline btuC & Iron $\mathrm{ABC}$ transporter permease & Rv2060 & 191.76 & 5.638421765 & 296.6266667 & 162.4777085 & 106.7166667 & 150.9201573 \\
\hline \multicolumn{9}{|c|}{ Anaerobic pathway } \\
\hline cbiX & Sirohydrochlorin ferrochelatase & Rv0259c & 12.73 & 1.498465882 & 0 & 0 & 0 & 0 \\
\hline \multicolumn{9}{|c|}{ Urpoporfirynogen III pathway } \\
\hline cysG & $\begin{array}{l}\text { Multifunctional uroporphyrin-III C-methyltransferase/ } \\
\text { precorrin-2 oxidase/ferrochelatase }\end{array}$ & Rv2847c & 68.92 & 5.318890862 & 36.77 & 10.84717782 & 63.26 & 89.46314996 \\
\hline cysH & Phosphoadenylyl-sulfate reductase & Rv2392 & 312.9333333 & 19.43125032 & 503.0566667 & 166.2576146 & 0 & 0 \\
\hline gltX & Glutamyl-tRNA synthetase & Rv2992c & 170.96 & 11.21727537 & 89.43333333 & 42.90810128 & 107.7533333 & 76.94248949 \\
\hline hemA & Glutamyl-tRNA reductase & Rv0509 & 612.7766667 & 65.26401271 & 698.94 & 222.6659401 & 362.77 & 513.034254 \\
\hline hemB & $\begin{array}{l}\text { Probable delta-aminolevulinic acid dehydratase/porpho- } \\
\text { bilinogen synthase }\end{array}$ & Rv0512 & 279.2466667 & 19.95736511 & 165.0033333 & 36.41366075 & 160.32 & 114.4782576 \\
\hline hemC & Porphobilinogen deaminase & Rv0510 & 627.0233333 & 64.43651415 & 435.5433333 & 115.7868324 & 161.2566667 & 114.1554689 \\
\hline hemD & Uroporphyrinogen III methyltransferase/synthase & Rv0511 & 640.4733333 & 56.28158037 & 342.2533333 & 64.04123949 & 138.85 & 9.488702054 \\
\hline hemL & Glutamate-1-semialdehyde 2,1-aminomutase & Rv0524 & 437.8066667 & 25.11909809 & 114.9666667 & 72.38097832 & 391.8 & 135.6539762 \\
\hline hemY & ChlI component of cobalt chelatase & Rv2850c & 135.66 & 6.78823001 & 38.01666667 & 3.454488224 & 81.53333333 & 115.3055458 \\
\hline \multicolumn{9}{|c|}{ Vitamin B12 dependent enzymes } \\
\hline metH & 5-Methyltetrahydrofolate-homocysteine methyltransferase & Rv2124c & 155.78 & 9.823003614 & 311.4966667 & 16.31473431 & 128.15 & 45.50090622 \\
\hline mutB & Methylmalonyl-CoA mutase & Rv1493 & 48.83 & 1.851323851 & 139.7433333 & 70.5155852 & 38.08333333 & 53.8579665 \\
\hline nrdZ & Ribonucleotide reductase of class II & Rv0570 & 60.79333333 & 1.360890232 & 366.2433333 & 120.7928051 & 119.6 & 101.265619 \\
\hline
\end{tabular}

Table 2. Expression of genes involved in cobalamin metabolism in M. tuberculosis H37Rv based on RNA-Seq data.

supplemented with cobalt to evade the blockade of synthesis due to insufficient cobalt concentration. First, we investigated the possibility of de novo synthesis of vitamin B12. We tested logarithmic phase cultures, stationary phase cultures, and acidified cultures that would mimic the infection's active stage. For granuloma conditions, we tested starved cultures, persister cultures, and hypoxic cultures. ELISA immunoassay detected less than one ng of vitamin B12 per one $\mathrm{ml}$ of lysate in all of the samples. The samples were considered negative for vitamin B12 based on the cut-off value of the sensitivity of the test. Taken that vitamin B12 tends to accumulate in the cells during prolonged growth, our results suggest that it is unlikely that there is an ongoing de novo synthesis of vitamin B12 inside M. tuberculosis cells.

Next, we wanted to see if $M$. tuberculosis might rely on substances widely present in the host to produce vita$\min \mathrm{B} 12$. We supplemented the growth medium with uroporphyrinogen III, which is a precursor of heme in the human body and a precursor of vitamin B12 in bacteria (Fig. 1). We tested cell lysates from logarithmic phase cultures, stationary phase cultures, persister cell cultures, and hypoxic cultures. ELISA immunoassay detected 
A)

B)

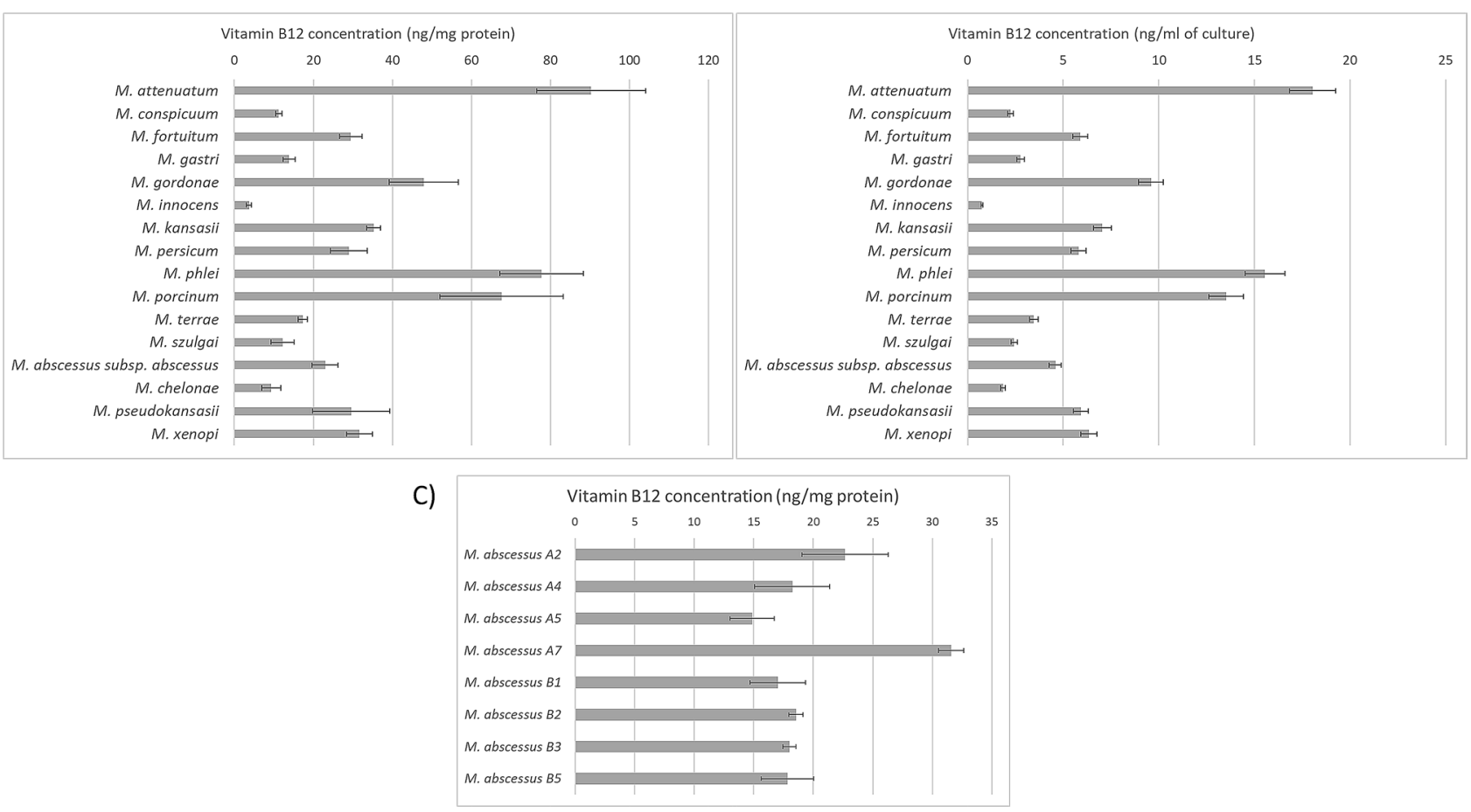

Figure 3. Cobalamin concentration in cell lysates of non-tuberculous mycobacteria. Cobalamin was detected in cell lysates of non-tuberculous mycobacteria by immunoassay. Cells were grown in 7H9 medium supplemented with $\mathrm{OADC}$, Tween 80 , and $\mathrm{CoCl}_{2}$. We cultured cells until the suspension reached $\mathrm{OD}_{600}=1$. Next, cells were harvested and washed with fresh medium without supplements to remove residual medium proteins from the surface. The pellet was re-suspended in Tris buffer and disrupted by beat-beating. The suspension was spinned. We used supernatant to estimate the concentration of vitamin B12 and protein content. Results were obtained from three independent cultures; each lysate was analyzed in two technical replicates. (A) cobalamin concentration in cell lysates of non-tuberculous mycobacteria, when normalized in ng per mg of protein, (B) cobalamin concentration in non-tuberculous mycobacteria cell lysates, when normalized in ng per ml of culture, (C) cobalamin concentration in cell lysates of clinical strains of M. abscessus complex. Whiskers represent SD.

less than one ng of vitamin B12 per one $\mathrm{ml}$ of lysate in all of the samples. Hence, the samples were considered negative for vitamin B12 based on the cut-off value of the test's sensitivity.

M. tuberculosis metE promoter responds to vitamin B12 concentration present in the host. We used the GFP reporter system to see if the cells of $M$. tuberculosis could respond to vitamin B12 concentration found within the host (Fig. 5). Vitamin B12 concentration in the human body is between 0.2 and $0.9 \mu \mathrm{g} / \mathrm{ml}$. We constructed a series of mutants, H37Rv::attB + rsB12, $\Delta b a c A:: a t t B+\mathrm{rsB} 12, \Delta c o b I J:: a t t B+\mathrm{rsB} 12$, carrying the gene of GFP under the control of the metE promoter, controlled by a vitamin B12-dependent riboswitch. In our model, the presence of vitamin B12 in the cells prevents translation of $g f p$ transcript, which results in diminished fluorescence. Of note, distinct clones of the same cell lines showed a different level of basal fluorescence without supplementation of medium without vitamin B12. Therefore, the fluorescence level could not be reliably compared between different cell lines due to the distinct basal expression of GFP in the clones. However, green fluorescence levels could be relatively compared within one clone of the cell line when considering different concentrations of vitamin B12 in the growth medium. We tested various concentrations of vitamin B12. We observed that supplementation of the growth medium with vitamin B12 gradually diminished gene expression of the green fluorescence protein of M. tuberculosis $\mathrm{H} 37 \mathrm{Rv}$ and $\Delta$ cobIJ from 100 to $23.93 \%$ and $23.70 \%$, respectively. In turn, the green fluorescence expression of $\triangle b a c A$ was not affected, and it remained constant at approximately $100 \%$. Similarly, the autofluorescence level was constant for the control strain M. tuberculosis H37Rv, which lacked the reporter system. M. tuberculosis H37Rv GFP expression diminished to $70.29 \%$ in the presence of $0.5 \mu \mathrm{g} / \mathrm{ml}$ of vitamin B12 $(p=0.01, \mathrm{t}=6.64, \mathrm{df}=3)$. Hence, $M$. tuberculosis metE promoter is responsive to vitamin B12 concentration found in the human body. Our results confirm the role of BacA as the transporter of vitamin $\mathrm{B} 12^{33}$. Further, our results indirectly confirm the lack of vitamin B12 production in M. tuberculosis $\mathrm{H} 37 \mathrm{Rv}$, because the wild type strain and the knock-out strain similarly showed a decrease in fluorescence corresponding to increasing concentration of supplemented vitamin B12.

Previous reports suggested that the ability to synthesize vitamin B12 by M. tuberculosis was restricted in $M$. cannetti like ancestor ${ }^{9}, 10$. M. tuberculosis is an obligate pathogen with possible access to vitamin B12 from the host. It is, therefore, possible that the genes involved in vitamin B12 synthesis in the genome of M. tuberculosis 


\section{RELATIVE GENE EXPRESSION DURING STARVATION}

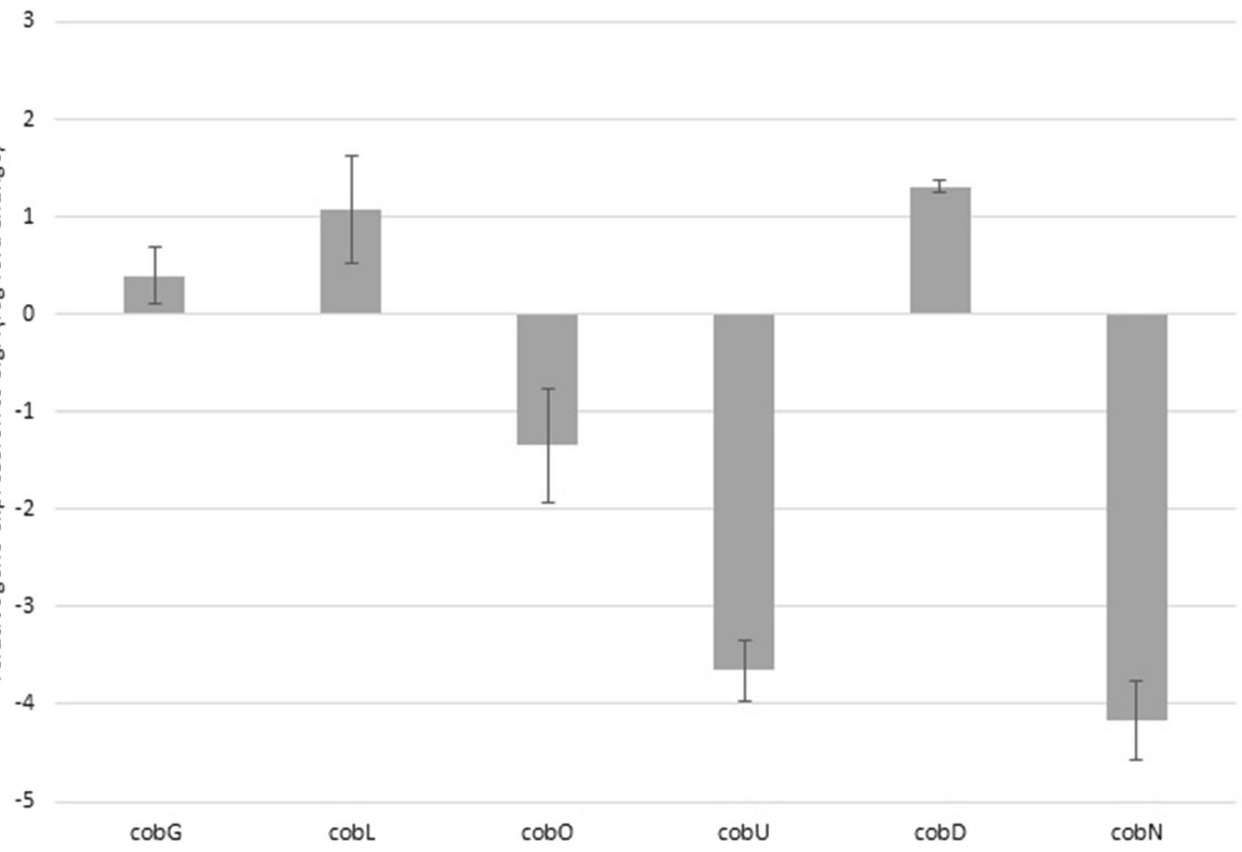

Figure 4. Relative gene expression of genes involved in cobalamin biosynthesis in M. smegmatis. Data across samples was normalized to sigA. Bars represent log fold change. Whiskers represent SD.

are remnants from a more independent ancestor. The genes of the vitamin B12 biosynthesis pathway in Mycobacterium leprae, another obligate pathogen of Mycobacterium genus, evolved into pseudogenes ${ }^{10}$. The most probable explanation is that M. tuberculosis does not synthesize vitamin B12 anymore. However, there was still not enough time since the abrogation of the pathway to accumulate mutations that would entirely degrade the pathway, impede the expression of genes and convert them into pseudogenes. It seems that the disruption of the pathway might have taken place relatively recently, as cobF encoding region was found in two $M$. tuberculosis strains found in the African Great Lakes region, representing Lineage 8 of $M$. tuberculosis complex ${ }^{31}$. To be precise, it cannot be excluded that M. tuberculosis does synthesize vitamin B12, but the level of vitamin concentration is undetectable by the immunoassay we used. Finally, it remains to be established whether M. tuberculosis is able to synthesize cobamides other than vitamin B12 $2^{34}$.

\section{Summary}

We conclude that mycobacteria are generally capable of vitamin B12 synthesis, with the likely exception of $M$. tuberculosis. Our results are direct evidence of vitamin B12 production in these clinically important group of bacteria.

\section{Materials and methods}

Bacterial strains. We analyzed the level of vitamin B12 in several type strains and clinical strains of nontuberculous mycobacteria (Table 4). Mycolicibacterium porcinum, M. fortuitum, and Mycobacteroides abscessus complex were isolated and differentiated in $\mathrm{Canada}^{35}$. Further, we included a laboratory strain of $M$. tuberculosis $\mathrm{H} 37 \mathrm{Rv}$, its genetically modified derivatives $\Delta c o b I J$ and $\Delta b a c A$, and five clinical strains of $M$. tuberculosis isolated in Lodz, Poland, between 2006 and 2008. Each strain belonged to a different clade. We chose strains: 321 (spoligotype 35, clade H4), 404 (spoligotype 46, clade U (likely H)), 663 (spoligotype 50, clade H3), 216/8 (spoligotype 42, clade LAM9) and 218/8 (spoligotype 1253, clade S) ${ }^{36}$. We used Escherichia coli Top10 for cloning.

Bacterial cultures. E. coli Top 10 . Bacteria were cultured at $37^{\circ} \mathrm{C}$ for $18-20 \mathrm{~h}$ in liquid or solid Luria-Bertani broth. Where necessary, the media were supplemented with antibiotics or other supplements at the following concentrations: kanamycin (Bioshop) $50 \mu \mathrm{g} / \mathrm{ml}$; ampicillin (Bioshop) $100 \mu \mathrm{g} / \mathrm{ml}$, X-gal $40 \mu \mathrm{g} / \mathrm{ml}$ (BioShop), sucrose $2 \%$ (Sigma Aldrich) at $37^{\circ} \mathrm{C}$.

Non-tuberculous and tuberculous mycobacteria. Where necessary, media were supplemented with $10 \%$ oleic acid albumin dextrose catalase growth supplement (OADC) (Becton-Dickinson), 0.05\% Tween 80 (Sigma), tyloxapol 0.015\% (Sigma-Aldrich), cobalt chloride $12 \mu \mathrm{g} / \mathrm{ml}$ (Sigma Aldrich), kanamycin $25 \mu \mathrm{g} / \mathrm{ml}$ (BioShop); X-gal $40 \mu \mathrm{g} / \mathrm{ml}$ (BioShop), sucrose 2\% (Sigma Aldrich), vitamin B12 (adenosylcobalamin) $10 \mu \mathrm{g} / \mathrm{ml}$ (Sigma Aldrich), uroporfirynogen III octamethyl ester $1 \mu \mathrm{g} / \mathrm{ml}$ (Sigma Aldrich) (dissolved in 25\% DMSO), OAD (0.05\% 


\begin{tabular}{|c|c|c|c|}
\hline Type of culture & Growth medium & Supplementation & Vitamin B12 \\
\hline Logarytmic phase cultures & $7 \mathrm{H} 9+\mathrm{OADC}+$ Tween $80+\mathrm{CoCl} 2$ & - & Negative \\
\hline Stationary phase cultures & 7H9+ OADC+ Tween $80+\mathrm{CoCl} 2$ & - & Negative \\
\hline Acidified cultures & 7H9+ OADC + Tween $80+\mathrm{CoCl} 2$, pH 5.5 & - & Negative \\
\hline Starved cultures & 7H9+ Tween $80+\mathrm{CoCl} 2$ & - & Negative \\
\hline Persister cultures & $\mathrm{K}+$ deficient Sauton medium $+\mathrm{CoCl} 2$ & - & Negative \\
\hline Hypoxic cultures & $7 \mathrm{H} 9+\mathrm{OAD}+$ Tween $80+\mathrm{CoCl} 2(+$ methylene blue), $25 \%$ head ratio & - & Negative \\
\hline Logarytmic phase cultures & $7 \mathrm{H} 9+\mathrm{OADC}+$ Tween $80+\mathrm{CoCl} 2$ & Uroporphyrinogen III & Negative \\
\hline Stationary phase cultures & $7 \mathrm{H} 9+\mathrm{OADC}+$ Tween $80+\mathrm{CoCl} 2$ & Uroporphyrinogen III & Negative \\
\hline Persister cultures & $\mathrm{K}+$ deficient Sauton medium $+\mathrm{CoCl} 2$ & Uroporphyrinogen III & Negative \\
\hline Hypoxic cultures & $7 \mathrm{H} 9+\mathrm{OAD}+$ Tween $80+\mathrm{CoCl} 2(+$ methylene blue), $25 \%$ head ratio & Uroporphyrinogen III & Negative \\
\hline
\end{tabular}

Table 3. Types of cultures tested for cobalamin in cells of $M$. tuberculosis $\mathrm{H} 37 \mathrm{Rv}$, five clinical strains of $M$. tuberculosis, and $\Delta$ cobIJ.

Relative green fluorescence value

1,4



Figure 5. The evaluation of the protein expression of $g f p$ gene under the control of vitamin B12- dependent riboswitch, based on the green fluorescence. We used a Guava flow cytometer to evaluate the green fluorescence in cells of H37Rv::attB + rsB12, $\triangle b a c A:: a t t B+$ rsB12, $\Delta$ cobIJ::attB + rsB12, carrying the gene of GFP under the control of the metE promoter, controlled by a vitamin B12-dependent riboswitch. We observed that the green fluorescence of strains H37Rv::attB + rsB12 and $\Delta$ cobIJ::attB + rsB12 significantly differed from the control strains grown in medium without exogenous vitamin B12 supplementation. Each strain was analyzed in samples collected from three cultures. Statistical analysis was performed with paired t-test. Whiskers represent SD.

Oleic Acid, $5 \%$ bovine serum albumin, fraction V, $2 \%$ glucose, and $0.85 \% \mathrm{NaCl}$ ). All cultures were started at $\mathrm{OD}_{600}=0.1$.

Unless stated otherwise, cultures and seed cultures of non-tuberculous mycobacteria and M. tuberculosis were cultivated in $7 \mathrm{H} 9$ broth supplemented with OADC, Tween 80 , and cobalt chloride. Cultures of non-tuberculous mycobacteria were started at OD600 $=0.05$ and carried out until they reached OD $600=1$. M. tuberculosis cultures were started at $\mathrm{OD} 600=0.1$. For seeding, the appropriate amount of logarithmic phase culture $(\mathrm{OD} 600=0.8)$ was spinned down, washed in fresh medium, spinned again, and re-suspended in fresh medium. 
For testing vitamin B12 concentration, non-tuberculous mycobacteria were cultured in 7H9 broth supplemented with OADC, Tween 80, and cobalt chloride until they reached OD600 $=1$. Starved cultures of $M$. smegmatis were cultured as described previously ${ }^{17}$.

We tested several types of cultures of M. tuberculosis for vitamin B12 concentration. Logarithmic phase cultures of M. tuberculosis were grown in 7H9 medium supplemented with OADC, Tween 80 , and cobalt chloride until they reached OD600 $=0.8$. Stationary phase cultures were carried out in the same medium, and they were collected after 15 days of culture. Acidified cultures ( $\mathrm{pH} 5.7$ ) were carried out in 7H9 broth, supplemented with $5 \%$ bovine serum albumin, fraction $\mathrm{V}, 2 \%$ glucose, and $0.85 \% \mathrm{NaCl}$, Tween 80 , and cobalt chloride. They were collected after 1 week of culture. Starved cultures were carried out in 7H9 broth supplemented with Tween 80, $0.5 \%$ glycerol, and cobalt chloride. They were collected after one week of culture. Hypoxic cultures were carried out as previously described ${ }^{37}$. In brief, starter cultures of $M$. tuberculosis grown on Dubos medium supplemented with OAD and cobalt chloride were tightly locked in flasks with 0.25 headspace ratio and cultured at $37^{\circ} \mathrm{C}$ on a shaker for six weeks. Catalase is not recommended for use in hypoxia experiments because it influences redox balance, and redox stress is an important stress factor of hypoxia ${ }^{38}$. Methylene blue was added to control cultures as an indicator of oxygen depletion. After this time, the flasks were opened, cultures were spinned down and washed three times with 7H9 medium. A sample of the culture was plated as viability control, while the rest was lyzed. For persister cultures, we used a medium deprived of $\mathrm{K}+$, as previously described ${ }^{16}$. Here, starter cultures grown on Sauton medium were spinned down and re-suspended in Sauton medium deficient in K+ supplemented with cobalt chloride. After two weeks, rifampicin was added to cultures at $5 \mu \mathrm{g} \mathrm{ml}^{-1}$ and the culture continued for the next four weeks at $37^{\circ} \mathrm{C}$.

Cloning strategy. All molecular cloning was performed in E. coli T10. Knock-out mutants of mycobacteria were obtained by the method of gene replacement through homologous recombination (Tables 5, 6; Fig. 6; Supplementary Figs. 1 and 2$)^{39}$. Briefly, sequences flanking desired deletion were amplified by PCR. We used AccuPrime Pfx High Fidelity Polymerase (Invitrogen), and genomic DNA of M. tuberculosis H37Rv for the reaction. PCR products were introduced into pJET1.2 plasmid (Thermo Fisher Scientific) and sequenced. Following confirmation of cloning of proper sequence, we cut out flanking sequences using restriction enzymes, and sequentially introduced them into p2NIL plasmid, together with marker genes from pGOAL17. Plasmids were transformed into M. tuberculosis $\mathrm{H} 37 \mathrm{Rv}$ thru electroporation. The cells underwent gene replacement by allelic exchange as described previously ${ }^{39}$. Similarly, episome plasmid containing green fluorescence protein (GFP) gene under the control of the riboswitch of $M$. tuberculosis metE gene was constructed with a similar procedure. We started with PCR amplification of products on genomic DNA of M. tuberculosis H37Rv and pJAM plasmid carrying $g f p$. Subsequently, we introduced sequences to pJET1.2, and we confirmed proper cloning by sequencing. Next, we used restriction digestion to cut out the sequences, and we introduced them into pMV306 episome plasmid. M. tuberculosis $\mathrm{H} 37 \mathrm{Rv}$ and its derivative strains were transformed by electroporation.

Vitamin B12 ELISA. Bacterial cultures were spinned down, washed with fresh medium without supplements, spinned down again, and re-suspended in 0.01 M TRIS pH 7.5. The mixture was transferred to disruptor eppendorfs. Cells were disrupted twice using the MP disruptor system with the Quick prep adapter (MP Biomedicals) and $0.1 \mathrm{~mm}$ silica spheres ( $45 \mathrm{~s}, 6.0 \mathrm{~m} / \mathrm{s}$ with $5 \mathrm{~min}$ intervals). Samples were spinned down, and lysates were transferred to new eppendorfs. As a principal, we normalized the results regarding vitamin B12 concentration to protein concentration in cell lysates. We wanted to avoid errors resulting from a different level of difficulty to disrupt mycobacterial cells of different species. Protein concentration in lysates was measured using Bradford reagent (BioShop) and estimated with a standard curve. In order to achieve a sufficient detection limit of vitamin B12, we only used the lysates that contained at least $0.5 \mathrm{mg}$ of protein per $\mathrm{ml}$, preferably between 1 and $2 \mathrm{mg}$ of protein per $\mathrm{ml}$.

Vitamin B12 ELISA (Demeditec) was performed according to manufacturer instructions. The test is based on the principle of the competitive enzyme-linked immunosorbent assay. The surface of a microtiter plate was covered with an antibody directed against vitamin B12 by the manufacturer. Samples and standards were mixed with a vitamin B12-peroxidase conjugate in the wells of the microtiter plate. Both enzyme-labeled and free vitamin B12 competed for the antibody binding sites. After one hour of incubation at room temperature, the wells were washed to remove the unbound material. A substrate solution was added, resulting in the development of a blue color. The color development was inhibited by the addition of a stop solution, and the color turned yellow. The yellow color was measured photometrically at $450 \mathrm{~nm}$. The concentration of vitamin B12 was indirectly proportional to the color intensity of the test sample.

For each condition, we analyzed lysates from three independent cultures. Each sample was analyzed in duplicate wells, as recommended by the producer of the immunoassay. The minimum detection level of vitamin B12 was settled at $1 \mathrm{ng} / \mathrm{ml}$ based on our previous observations ${ }^{17}$, and all samples below this level were considered negative for vitamin B12. For purposes of enabling comparison with the results obtained from other species of bacteria, our results were also normalized to $\mathrm{ml}$ of culture.

Flow cytometer analysis. Samples of cultures were analyzed on the flow cytometer Guava EasyCyte Flow Cytometer with High Power Blue Laser (Merck) suitable for detection of bacteria. Unstained control samples were diluted to reach a concentration of 400-800 cells/ $\mu$ l. Cell suspensions were first run through the flow cytometer to set a population gate around the bacteria by using the forward-scatter versus side-scatter parameters. Next, the voltages in the green fluorescence channel were adjusted so that the fluorescence histogram of the unstained bacteria appeared within the first compartments of the logarithmic scale of fluorescence. Ten 


\begin{tabular}{|c|c|c|}
\hline Species & Strain & Description \\
\hline \multicolumn{3}{|l|}{ Non-tuberculous mycobacteria } \\
\hline M. smegmatis & mc2 & Type strain \\
\hline M. attenuatum & DSM 107153 & Type strain \\
\hline M. chelonae & ATCC 35752 & Type strain \\
\hline M. conspicuum & DSM 44136 & Type strain \\
\hline M. gastrii & DSM 43505 & Type strain \\
\hline M. innocens & DSM 107161 & Type strain \\
\hline M. kansasii & ATCC12478 & Type strain \\
\hline M. persicum & DSM 104278 & Type strain \\
\hline M. phlei & JCM 5865 & Type strain \\
\hline M. pseudokansasii & DSM 107152 & Type strain \\
\hline M. szulgai & DSM 44166 & Type strain \\
\hline M. terrae & JCM 12143 & Type strain \\
\hline M. fortuitum & F1 & Clinical isolate \\
\hline M. gordonae & G1 & Clinical isolate \\
\hline M. porcinum & P1 & Clinical isolate \\
\hline M. xenopi & $\mathrm{X} 1$ & Clinical isolate \\
\hline M. abscessus subsp. abscessus & A2 & Clinical isolate \\
\hline M. abscessus subsp. abscessus & A4 & Clinical isolate \\
\hline M. abscessus subsp. abscessus & A5 & Clinical isolate \\
\hline M. abscessus subsp. abscessus & A7 & Clinical isolate \\
\hline M. abscessus subsp. bolletii & B1 & Clinical isolate \\
\hline M. abscessus subsp. bolletii & B2 & Clinical isolate \\
\hline M. abscessus subsp. bolletii & B3 & Clinical isolate \\
\hline M. abscessus subsp. bolletii & B5 & Clinical isolate \\
\hline \multicolumn{3}{|l|}{ M. tuberculosis } \\
\hline M. tuberculosis & H37Rv & Type strain, wild type \\
\hline M. tuberculosis & 321 & Clinical isolate \\
\hline M. tuberculosis & 404 & Clinical isolate \\
\hline M. tuberculosis & 663 & Clinical isolate \\
\hline M. tuberculosis & $216 / 8$ & Clinical isolate \\
\hline M. tuberculosis & $218 / 8$ & Clinical isolate \\
\hline \multicolumn{3}{|l|}{ Genetically modified strains } \\
\hline M. tuberculosis & SCO:bacA & Single cross over mutant \\
\hline M. tuberculosis & SCO:cobIJ & Single cross over mutant \\
\hline M. tuberculosis & $\triangle b a c A$ & Deletion mutant \\
\hline M. tuberculosis & $\Delta$ & Deletion mutant \\
\hline M. tuberculosis & H37Rv::attB + rsB12 & Wild type complemented with reporter system \\
\hline M. tuberculosis & $\Delta b a c A:: a t t B+\mathrm{rsB} 12$ & Mutant complemented with reporter system \\
\hline M. tuberculosis & $\Delta$ cobIJ::attB+rsB12 & Mutant complemented with reporter system \\
\hline
\end{tabular}

Table 4. List of strains used in this study.

thousand events were collected at a set standard low event rate. We used Guava software to analyze the acquired data. For each strain, we analyzed data from three cultures.

qPCR. Bacterial cultures were spinned down, re-suspended in water, and three volumes of TriReagent was added (Bioshop). The mixture was transferred to disruptor eppendorfs. Cells were disrupted twice using the MP disruptor system with the Quick prep adapter (MP Biomedicals) and $0.1 \mathrm{~mm}$ silica spheres $(45 \mathrm{~s}, 6.0 \mathrm{~m} / \mathrm{s}$ with 5 min intervals). Samples were spinned down, and the supernatant was transferred to new eppendorfs. One volume of chloroform was added, samples were vigorously mixed and spinned down. The top phase was transferred to new eppendorfs and precipitated with 1 volume of isopropanol and 1/10 volume of sodium acetate. Following precipitation, samples were re-suspended in water and digested with Turbo DNase I (Invitrogen by Thermo Fisher Scientific) following the manufacturer's instructions. The RNA quantity was assessed using a NanoDrop 2000 spectrophotometer (Thermo Fisher Scientific). cDNA was obtained using SuperScript III First-Strand Synthesis Super Mix kit with random hexamers (Invitrogen). qPCR was performed using SG qPCR Master Mix $(2 \times)$, plus ROX Solution (Eurx), and synthetic primers (Table 5) on a 7900HT real-time PCR system (Applied Biosystems). Real-time PCR conditions were as follows: initial activation at $95{ }^{\circ} \mathrm{C}$ for $10 \mathrm{~min}$, followed by 40 


\begin{tabular}{|c|c|c|}
\hline Name & Primer orientation & Primer sequence $5^{\prime}$ \\
\hline \multicolumn{3}{|l|}{ Gene replacement } \\
\hline \multirow{2}{*}{$b a c A$ first flank } & Forward & CAGTACTAGGTTGGATCGGCGTGGATAAGC \\
\hline & Reverse & CAAGCTTCACAGATGGCACTGATCGTCCAGG \\
\hline \multirow{2}{*}{$b a c A$ second flank } & Forward & CAAGCTTGGCGAGCGGGTGGAAGGTACC \\
\hline & Reverse & CGGTACCAATACCGCCCACCCCACC \\
\hline \multirow{2}{*}{ cobIJ first flank } & Forward & CAGTACTGCGACCCATTCTCCCGTACG \\
\hline & Reverse & CAAGCTTCGTGTGGGGCGCTGTGATAG \\
\hline \multirow{2}{*}{ cobIJ second flank } & Forward & CAAGCTTGACTGGATGACACCGCAGAGCC \\
\hline & Reverse & CGGTACCATCACCTGGCAGATCCGCG \\
\hline \multicolumn{3}{|l|}{ Gene complementation } \\
\hline \multirow{2}{*}{ metE promoter region } & Forward & CGGTACCCTCGGGAACCGGCTTTAACACGG \\
\hline & Reverse & CTCTAGAGGTGTTCACCGGCACCGAGTCC \\
\hline \multirow{2}{*}{ Green fluorescence protein } & Forward & CTCTAGAATGAGTAAAGGAGAAGAACTTTTCACTGG \\
\hline & Reverse & CAAGCTTCTATTTGTATAGTTCATCCATGCCATGTG \\
\hline \multicolumn{3}{|l|}{ Southern blot } \\
\hline \multirow{2}{*}{ bacA probe } & Forward & GCGGCGAGAACGAGACGATG \\
\hline & Reverse & CGCCACCGAGTAGTTCGAGCTG \\
\hline \multirow{2}{*}{ cobIJ probe } & Forward & ATGAGCGCTCGGGGCACGC \\
\hline & Reverse & TCAGTCGCTGTGGCGGCTCG \\
\hline \multicolumn{3}{|l|}{ qPCR } \\
\hline \multirow{2}{*}{$\operatorname{cobG}$} & Forward & CGCTCGTGTGTCGGTGACGG \\
\hline & Reverse & AGTGCACCAGGCCGCTGACG \\
\hline \multirow{2}{*}{$c o b L$} & Forward & ACGCGCGACCGTGGTGTTC \\
\hline & Reverse & TCGACACGTGCGGCAGCA \\
\hline \multirow{2}{*}{$c o b O$} & Forward & TCGTCGCTGCCGTGTTTGC \\
\hline & Reverse & GGCGTTCGGGATGGCGTT \\
\hline \multirow{2}{*}{$c o b U$} & Forward & ACGGTCTGCCAGTGTGCGGG \\
\hline & Reverse & CCGGGAAATCGCAGTGGGC \\
\hline \multirow{2}{*}{$\operatorname{cob} D$} & Forward & TGGCGCTGTTCGGTTCCGG \\
\hline & Reverse & CCAGGTGTGGGCGGTTTCTGC \\
\hline \multirow{2}{*}{$c o b N$} & Forward & GTGGTCAGCGGCGAGCAGAC \\
\hline & Reverse & AGGGGGCGTTCGAGGATGC \\
\hline \multirow{2}{*}{$\operatorname{sig} A$} & Forward & AGAAAGCCCCGGCCAAGCG \\
\hline & Reverse & GCGTCGCGGCATCAGCTTCT \\
\hline \multicolumn{3}{|c|}{ PCR confirmation of gene complementation } \\
\hline \multirow{2}{*}{ pMV306 } & Forward & GTGGATAACCGTATTACCGC \\
\hline & Reverse & AAGGCCCAGTCTTTCGACTGAG \\
\hline
\end{tabular}

Table 5. List of primers used in this study.

cycles at $94{ }^{\circ} \mathrm{C}$ for $15 \mathrm{~s}$ (denaturation), $62{ }^{\circ} \mathrm{C}$ for $30 \mathrm{~s}$ (annealing), $72{ }^{\circ} \mathrm{C}$ for $30 \mathrm{~s}$ (extension). The melting curve analysis was performed at the end of each qPCR reaction to verify a single, specific product was generated. The threshold cycle $(\mathrm{CT})$ value for each studied gene was normalized to the expression of msmeg_2758 $(\operatorname{sig} A)(\Delta \mathrm{CT})$ and converted to linear form $\left(2-\Delta \mathrm{C}_{\mathrm{T}}\right)$. The RNA samples for each strain were isolated from three independently grown cultures. Each sample for qPCR was run in triplicate.

RNA Seq. $\quad$ Raw RNA Seq reads were downloaded from European Nucleotide Archive Database (ENA). We analyzed gene expression of $M$. abscessus subsp. abscessus grown in 7H9 medium supplemented with OADC ${ }^{40}$, M. smegmatis grown in 7H9 medium with glucose $\mathrm{e}^{41}$, and three experiments performed with $M$. tuberculosis $\mathrm{H} 37 \mathrm{Rv}$ grown in $7 \mathrm{H} 9$ broth supplemented with $\mathrm{OADC}^{20}$, in a medium supplemented with cholesterol as a sole carbon source ${ }^{21}$ and in human THP-1 derived macrophages three days post-infection. Each experiment contained data for three replicates. Raw sequences were uploaded and processed with Geneious Prime 2021 (Biomatters, New Zealand). Reads were mapped to M. tuberculosis H37Rv accession number NC_000962 using Bowtie2 Geneious plug-in ${ }^{42}$. Gene expression analysis, through estimation of transcripts per kilobase million (TPM), was performed with Geneious. 


\begin{tabular}{|c|c|c|}
\hline Name & Description & Source \\
\hline pJET1.2 & Commercial plasmid & Thermo Fisher Scientific \\
\hline pJAM + gfp & pJAM plasmid carrying green fluorescence protein gene & Institute of Medical Biology \\
\hline p2NIL & \multirow{2}{*}{ Recombination vector, nonreplicating in mycobacteria } & Parish and Stocker, 2000 \\
\hline pGOAL17 & & Parish and Stocker, 2000 \\
\hline pMV306 & Mycobacterial integrating vector & Med-Immune Inc \\
\hline pAM1 & pJET1.2 carrying first flank of cobIJ gene & This study \\
\hline pAM2 & pJET1.2 carrying second flank of cobIJ gene & This study \\
\hline pAM3 & p2NIL carrying first flank of cobIJ gene & This study \\
\hline pAM4 & p2NIL carrying first and second flank of cobIJ gene & This study \\
\hline pAM5 & $\begin{array}{l}\text { p2NIL plasmid carrying flanking sequences of deletion within cobIJ gene, and marker genes } \\
\text { of pGOAL17 plasmid, KmR, lacZ+ }\end{array}$ & This study \\
\hline $\mathrm{pAB1}$ & pJET1.2 carrying first flank of bacA gene & This study \\
\hline pAB2 & pJET1.2 carrying second flank of bacA gene & This study \\
\hline pAB3 & p2NIL carrying first flank of bacA gene & This study \\
\hline pAB4 & p2NIL carrying first and second flank of bacA gene & This study \\
\hline pAB5 & $\begin{array}{l}\text { p2NIL plasmid carrying flanking sequences of deletion within bacA gene, and marker genes } \\
\text { of pGOAL17 plasmid, KmR, lacZ+ }\end{array}$ & This study \\
\hline pAM6 & pJET1.2 carrying metE promoter region & This study \\
\hline pAM7 & pJET1.2 carrying gfp & This study \\
\hline pAM8 & pMV306 carrying metE promoter region & This study \\
\hline pAM9 & $\begin{array}{l}\text { pMV306 plasmid carrying green fluorescence protein (GFP) gene under the control of the } \\
\text { riboswitch of } M \text {. tuberculosis metE gene }\end{array}$ & This study \\
\hline
\end{tabular}

Table 6. List of plasmids used in this study.

A)


1. M. tuberculosis H37Rv

2. M. tuberculosis SCO:coblJ clone 1

3. M. tuberculosis SCO:coblJ clone 2

4. M. tuberculosis $\Delta$ coblJ
B)

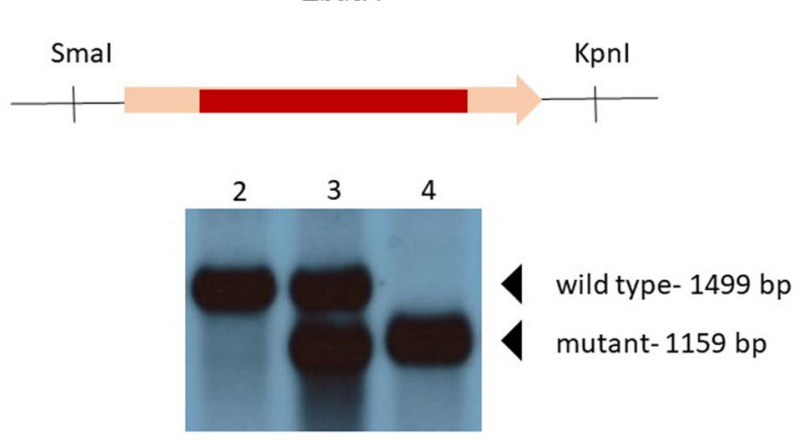

Figure 6. Southern blots confirming deletion of genes cobIJ and bacA in M. tuberculosis H37Rv. We used a gene replacement method through homologous recombination to obtain unmarked genetic mutants with large deletions inside the genes. Single cross-over (SCO) describes an intermediate step of mutagenesis. The images are cropped, hence altered lane numbering. Full-size images can be found in the supplementary data.

Identification of loci in the whole genome sequencing data based on the genome annotation. We identified genes involved in vitamin B12 metabolism in the following strains: M. tuberculosis $\mathrm{H} 37 \mathrm{Rv}$ (NC_000962), M. abscessus subsp. abscessus ATCC19977 (NC_010397), M. abscessus subsp. bolletii FLAC 003 (CP014950), M. conspicuum JCM 14738 (GCA_010730195), M. fortuitum CT6 (CP011269), M. gastri DSM 43505 (LQOX1000000), M. gordonae 24T (CP059165), M. innocens MK13 (LS999933), M. kansasii ATCC 12478 (GCA_000157895.1), M. persicum H48 (GCA_002705835), M. phlei CCUG 21000 (GCA_001582015), M. porcinum ACS 3670 (NZ_MBDY01000007.1), M. terrae NCTC 10856 (GCA_900187145), M. xenopi RIVM700366 (NZ_AJFI01000095.1), M. szulgai DSM 44166 (NZ_LQPW01000016.1) and M. smegmatis mc ${ }^{2} 155$ (CP009494). We screened the following databases: National Center of Biotechnology Information, Nucleotide and Protein 
(NCBI), Mycobrowser, STRING, UniProt, and we manually screened the sequences thru Geneious Prime (Biomatters, New Zealand).

Statistical analysis. Statistical analysis was performed with Develve Statistical Software, with paired t-test. The level of statistical significance was $p<0.05$. All results are reported as the means \pm SD unless otherwise stated.

Received: 17 February 2021; Accepted: 26 May 2021

Published online: 10 June 2021

\section{References}

1. Acevedo-Rocha, C. G., Gronenberg, L. S., Mack, M., Commichau, F. M. \& Genee, H. J. Microbial cell factories for the sustainable manufacturing of B vitamins. Curr. Opin. Biotechnol. 56, 18-29 (2019).

2. Fang, H., Kang, J. \& Zhang, D. Microbial production of vitamin B12: A review and future perspectives. Microb. Cell Fact 16, 15 (2017).

3. Chimento, D. P., Kadner, R. J. \& Wiener, M. C. The Escherichia coli outer membrane cobalamin transporter BtuB: Structural analysis of calcium and substrate binding, and identification of orthologous transporters by sequence/structure conservation. J. Mol. Biol. 332, 999-1014 (2003).

4. Domenech, P., Kobayashi, H., LeVier, K., Walker, G. C. \& Barry, C. E. BacA, an ABC transporter involved in maintenance of chronic murine infections with Mycobacterium tuberculosis. J. Bacteriol. 191, 477-485 (2009).

5. Larsson, K.-M., Logan, D. T. \& Nordlund, P. Structural basis for adenosylcobalamin activation in AdoCbl-dependent ribonucleotide reductases. ACS Chem. Biol. 5, 933-942 (2010).

6. Dorweiler, J. S., Finke, R. G. \& Matthews, R. G. Cobalamin-dependent methionine synthase: Probing the role of the axial base in catalysis of methyl transfer between methyltetrahydrofolate and exogenous cob(I)alamin or cob(I)inamide. Biochemistry 42, 14653-14662 (2003)

7. Banerjee, R. \& Ragsdale, S. W. The many faces of vitamin B12: Catalysis by cobalamin-dependent enzymes. Annu. Rev. Biochem. 72, 209-247 (2003).

8. Serganov, A. \& Nudler, E. A decade of riboswitches. Cell 152, 17-24 (2013).

9. Supply, P. et al. Genomic analysis of smooth tubercle bacilli provides insights into ancestry and pathoadaptation of Mycobacterium tuberculosis. Nat. Genet. 45, 172-179 (2013).

10. Young, D. B., Comas, I. \& de Carvalho, L. P. S. Phylogenetic analysis of vitamin B12-related metabolism in Mycobacterium tuberculosis. Struct. Biol. 2, 6 (2015).

11. Warner, D. F., Savvi, S., Mizrahi, V. \& Dawes, S. S. A riboswitch regulates expression of the coenzyme B12-independent methionine synthase in Mycobacterium tuberculosis: Implications for differential methionine synthase function in strains H37Rv and CDC1551. J. Bacteriol. 189, 3655-3659 (2007).

12. Gopinath, K. et al. A vitamin B12 transporter in Mycobacterium tuberculosis. Open Biol. https://doi.org/10.1098/rsob.120175 (2013).

13. Savvi, S. et al. Functional characterization of a vitamin B12-dependent methylmalonyl pathway in Mycobacterium tuberculosis: Implications for propionate metabolism during growth on fatty acids. J. Bacteriol. 190, 3886-3895 (2008).

14. Guzzo, M. B. et al. Methylfolate trap promotes bacterial thymineless death by sulfa drugs. PLoS Pathog. 12, e1005949 (2016).

15. Minias, A., Minias, P., Czubat, B. \& Dziadek, J. Purifying selective pressure suggests the functionality of a vitamin B12 biosynthesis pathway in a global population of Mycobacterium tuberculosis. Genome Biol. Evol. https://doi.org/10.1093/gbe/evy153 (2018).

16. Ignatov, D. V. et al. Dormant non-culturable Mycobacterium tuberculosis retains stable low-abundant mRNA. BMC Genomics 16, 954 (2015).

17. Czubat, B. et al. Functional disassociation between the protein domains of MSMEG_4305 of Mycolicibacterium smegmatis (Mycobacterium smegmatis) in vivo. Front. Microbiol. 11, 2008 (2020).

18. Kipkorir, T. et al. De novo cobalamin biosynthesis, transport and assimilation and cobalamin-mediated regulation of methionine biosynthesis in Mycobacterium smegmatis. J. Bacteriol. https://doi.org/10.1128/JB.00620-20 (2021).

19. Karasseva, V., Weiszfeiler, J. G. \& Lengyel, Z. Synthesis of vitamin B12 by various species of mycobacteria. Zentralbl Bakteriol Orig. A 239, 514-520 (1977).

20. Płociński, P. et al. Proteomic and transcriptomic experiments reveal an essential role of RNA degradosome complexes in shaping the transcriptome of Mycobacterium tuberculosis. Nucleic Acids Res. 47, 5892-5905 (2019).

21. Talwar, S. et al. Role of VapBC12 toxin-antitoxin locus in cholesterol-induced mycobacterial persistence. $m S y s t e m s ~ 5$, e00855 (2020).

22. Coskun, F. S. et al. sncRNA-1 is a small noncoding RNA produced by mycobacterium tuberculosis in infected cells that positively regulates genes coupled to oleic acid biosynthesis. Front. Microbiol. 11, 1631 (2020).

23. Kuron, A. et al. Evaluation of DNA primase DnaG as a potential target for antibiotics. Antimicrob. Agents Chemother. 58, 1699-1706 (2014).

24. Li, J., Ge, Y., Zadeh, M., Curtiss, R. \& Mohamadzadeh, M. Regulating vitamin B12 biosynthesis via the cbiMCbl riboswitch in propionibacterium strain UF1. PNAS 117, 602-609 (2020).

25. Nahvi, A., Barrick, J. E. \& Breaker, R. R. Coenzyme B12 riboswitches are widespread genetic control elements in prokaryotes. Nucleic Acids Res. 32, 143-150 (2004).

26. Pisu, D., Huang, L., Grenier, J. K. \& Russell, D. G. Dual RNA-Seq of Mtb-infected macrophages in vivo reveals ontologically distinct host-pathogen interactions. Cell Rep. 30, 335-350.e4 (2020).

27. Bachmann, N. L. et al. Key transitions in the evolution of rapid and slow growing mycobacteria identified by comparative genomics. Front. Microbiol. 10, 3019 (2020).

28. Crespo, A., Blanco-Cabra, N. \& Torrents, E. Aerobic vitamin B12 biosynthesis is essential for Pseudomonas aeruginosa class II ribonucleotide reductase activity during planktonic and biofilm growth. Front. Microbiol. 9, 986 (2018).

29. Deptula, P. et al. Food-like growth conditions support production of active vitamin B12 by Propionibacterium freudenreichii 2067 without DMBI, the lower ligand base, or cobalt supplementation. Front. Microbiol. 8, 368 (2017).

30. Arnold, F. H. Design by directed evolution. Acc. Chem. Res. 31, 125-131 (1998).

31. Ngabonziza, J. C. S. et al. A sister lineage of the Mycobacterium tuberculosis complex discovered in the African Great Lakes region. Nat. Commun. 11, 2917 (2020).

32. Reyrat, J. M. \& Kahn, D. Mycobacterium smegmatis: an absurd model for tuberculosis?. Trends Microbiol. 9, 472-474 (2001).

33. Rempel, S. et al. A mycobacterial ABC transporter mediates the uptake of hydrophilic compounds. Nature 580, 409-412 (2020).

34. Sokolovskaya, O. M., Shelton, A. N. \& Taga, M. E. Sharing vitamins: Cobamides unveil microbial interactions. Science 369, eaba0165 (2020). 
35. Minias, A. et al. Subspecies-specific sequence detection for differentiation of Mycobacterium abscessus complex. Sci. Rep. 10, 16415 (2020).

36. Krawczyk, M. et al. Epidemiological analysis of Mycobacterium tuberculosis strains isolated in Lodz. Poland. Int. J. Tuberc. Lung Dis. 15, 1252-1258 (2011).

37. Tan, M. P. et al. Nitrate respiration protects hypoxic Mycobacterium tuberculosis against acid- and reactive nitrogen species stresses. PLoS One 5, e13356 (2010).

38. Mavi, P. S., Singh, S. \& Kumar, A. Reductive stress: new insights in physiology and drug tolerance of mycobacterium. Antioxid. Redox Signal 32, 1348-1366 (2020).

39. Parish, T. \& Stoker, N. G. Use of a flexible cassette method to generate a double unmarked Mycobacterium tuberculosis tlyA plcABC mutant by gene replacement. Microbiology (Reading, England) 146(Pt 8), 1969-1975 (2000).

40. Miranda-CasoLuengo, A. A., Staunton, P. M., Dinan, A. M., Lohan, A. J. \& Loftus, B. J. Functional characterization of the $M y c o-$ bacterium abscessus genome coupled with condition specific transcriptomics reveals conserved molecular strategies for host adaptation and persistence. BMC Genomics 17, 553 (2016).

41. Oh, Y. et al. The partner switching system of the SigF sigma factor in Mycobacterium smegmatis and induction of the SigF regulation under respiration-inhibitory conditions. Front. Microbiol. 11, 588487 (2020).

42. Langmead, B. \& Salzberg, S. L. Fast gapped-read alignment with Bowtie 2. Nat. Methods 9, 357-359 (2012)

\section{Acknowledgements}

This work was performed within the FATE research consortium (https://fate-consortium.org/). This work is part of the research project financed by the National Science Center of Poland, Grant No. 2015/19/D/NZ6/03011. We are grateful to Heather Adam for providing strains for this research.

\section{Author contributions}

A.M. and J.D. designed the study. A.M., F.G., A.B. carried out the experiments. T.J. provided strains. A.M. wrote the manuscript. F.G., T.J., and J.D. corrected the manuscript. A.M. and J.D. supervised the project.

\section{Competing interests}

The authors declare no competing interests.

\section{Additional information}

Supplementary Information The online version contains supplementary material available at https://doi.org/ 10.1038/s41598-021-91430-w.

Correspondence and requests for materials should be addressed to A.M. or J.D.

Reprints and permissions information is available at www.nature.com/reprints.

Publisher's note Springer Nature remains neutral with regard to jurisdictional claims in published maps and institutional affiliations.

(c) (i) Open Access This article is licensed under a Creative Commons Attribution 4.0 International

License, which permits use, sharing, adaptation, distribution and reproduction in any medium or format, as long as you give appropriate credit to the original author(s) and the source, provide a link to the Creative Commons licence, and indicate if changes were made. The images or other third party material in this article are included in the article's Creative Commons licence, unless indicated otherwise in a credit line to the material. If material is not included in the article's Creative Commons licence and your intended use is not permitted by statutory regulation or exceeds the permitted use, you will need to obtain permission directly from the copyright holder. To view a copy of this licence, visit http://creativecommons.org/licenses/by/4.0/.

(c) The Author(s) 2021 